\title{
20. RADIOLARIA FROM THE NORTHEASTERN ATLANTIC OCEAN DSDP LEG 48
}

\author{
Annika Sanfilippo and W.R. Riedel, \\ Scripps Institution of Oceanography, La Jolla, California
}

\begin{abstract}
This cruise collected sequences of radiolarian samples through the middle and early Eocene, which provide useful standards of comparison for other North Atlantic assemblages of this age. Two new species are described - Pterocodon lex and Lamptonium obelix. A few Neogene, Paleocene, and Cretaceous samples yielded identifiable radiolarians.
\end{abstract}

\section{INTRODUCTION}

During Leg 48 Glomar Challenger carried no radiolarian paleontologist, and therefore the samples (many of them from core catchers), on which this chapter was based, were taken and sent to us by members of the shipboard party.

Samples from the following sites yielded radiolarians: Site $400-47^{\circ} 22.90^{\prime} \mathrm{N}, 09^{\circ} 11.90^{\prime} \mathrm{W}$; water depth 4399 meters

Site $401-47^{\circ} 25.65^{\prime} \mathrm{N}, 08^{\circ} 48.62^{\prime} \mathrm{W}$; water depth 2495 meters

Site $402-47^{\circ} 52.48^{\prime} \mathrm{N}, 08^{\circ} 50.44^{\prime} \mathrm{W}$; water depth 2339.5 meters

Site 403 - $56^{\circ} 08.13^{\prime} \mathrm{N}, 23^{\circ} 17.64^{\prime} \mathrm{W}$; water depth 2301 meters

Site $404-56^{\circ} 03.13^{\prime} \mathrm{N}, 23^{\circ} 14.95^{\prime} \mathrm{W}$; water depth 2306 meters

Site 405 - $55^{\circ} 20.18^{\prime} \mathrm{N}, 22^{\circ} 03.49^{\prime} \mathrm{W}$; water depth 2958 meters

For the sites at which there are considerable numbers of samples with radiolarians adequate for stratigraphic interpretation, species present in each sample are tabulated (Tables 1 and 2). For other sites, a paragraph describes the occurrences in general terms. Table 3 is a list of radiolarian events (earliest and latest occurrences of taxa) arranged stratigraphically, from which is derived the summary range chart of Figure 2. In Figure 3, Eocene sections from Leg 48 are correlated with one another and with a low-latitude section cored on DSDP Leg 10. There is no systematic section, its place being taken by a species list which includes, however, descriptions of two new taxa.

\section{OCCURRENCES OF RADIOLARIANS}

The following paragraphs briefly describe the radiolarian occurrences, Figure 1 summarizes this information in the framework of age assignments given in the Initial Core Description for Leg 48, and zonal assignments for the Eocene samples are indicated in Section 3 on stratigraphic correlation.

\section{Hole 400A}

Radiolarians of generally poor preservation occur in varying abundance in the Cretaceous to middle Eocene, and the Oligocene through Miocene parts of the section.
Assemblages in the late Miocene samples are too sparse and corroded to be useful for stratigraphic or taxonomic interpretation, with the slight exception of 400A-24, CC, which contains orosphaerid fragments (rare), actinommids (rare), pyloniids (rare), spyrids (rare), Eucyrtidium spp. (rare), Stichocorys delmontensis (rare), Carpocanistrum $\mathrm{sp}(\mathrm{p})$. (rare), Anthocyrtidium sp. (rare), Lamprocyclas sp. (rare), Lithomitra lineata group (rare), and Siphocampe corbula (rare).

The middle Miocene core catcher sample of Core 29 does not contribute to our taxonomic understanding, but it contains orosphaerid fragments (common), actinommids (rare), Cyrtocapsella japonica (rare), pterocorythid fragments (very rare), artostrobiid fragments (very rare).

Radiolarians in early Miocene and Oligocene samples through $400 \mathrm{~A}-45, \mathrm{CC}$ are too sparse and corroded to provide new taxonomic insight, but some stratigraphic hints are provided by the few forms indicated in Table 1 .

Although the radiolarians in $400 \mathrm{~A}-46, \mathrm{CC}$ are poorly preserved, they are certainly sufficient for interpretation as late Eocene rather than the early Oligocene indicated in the Initial Core Description. Species occurrences are shown in Table 2, and the correlation with other Eocene samples shown in Figure 3.

The radiolarians in middle and early Eocene samples from $400 \mathrm{~A}-47, \mathrm{CC}$ through $400 \mathrm{~A}-53, \mathrm{CC}$ are not well pre-

TABLE 1

Radiolarian Occurrences in Hole 400A

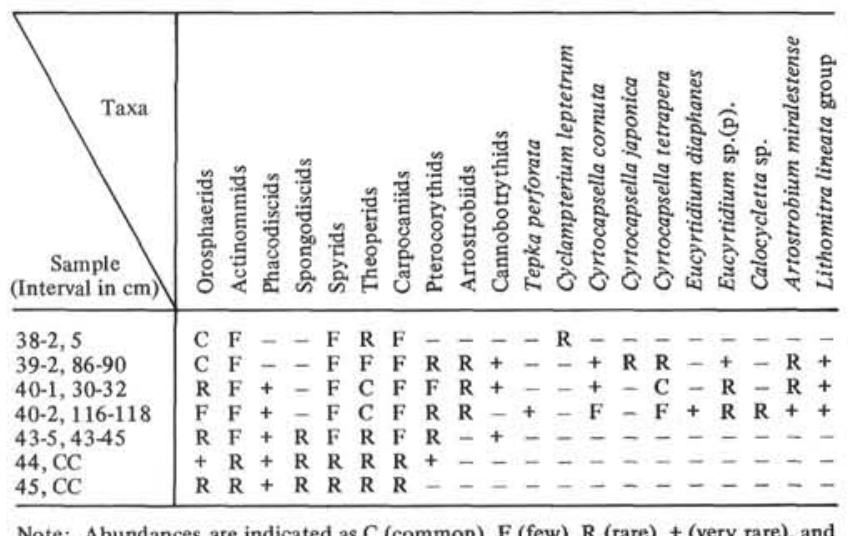

- (searched for but not found). 
TABLE 2

Eocene Radiolarian Occurrences at Sites 400, 401, 401, and 405

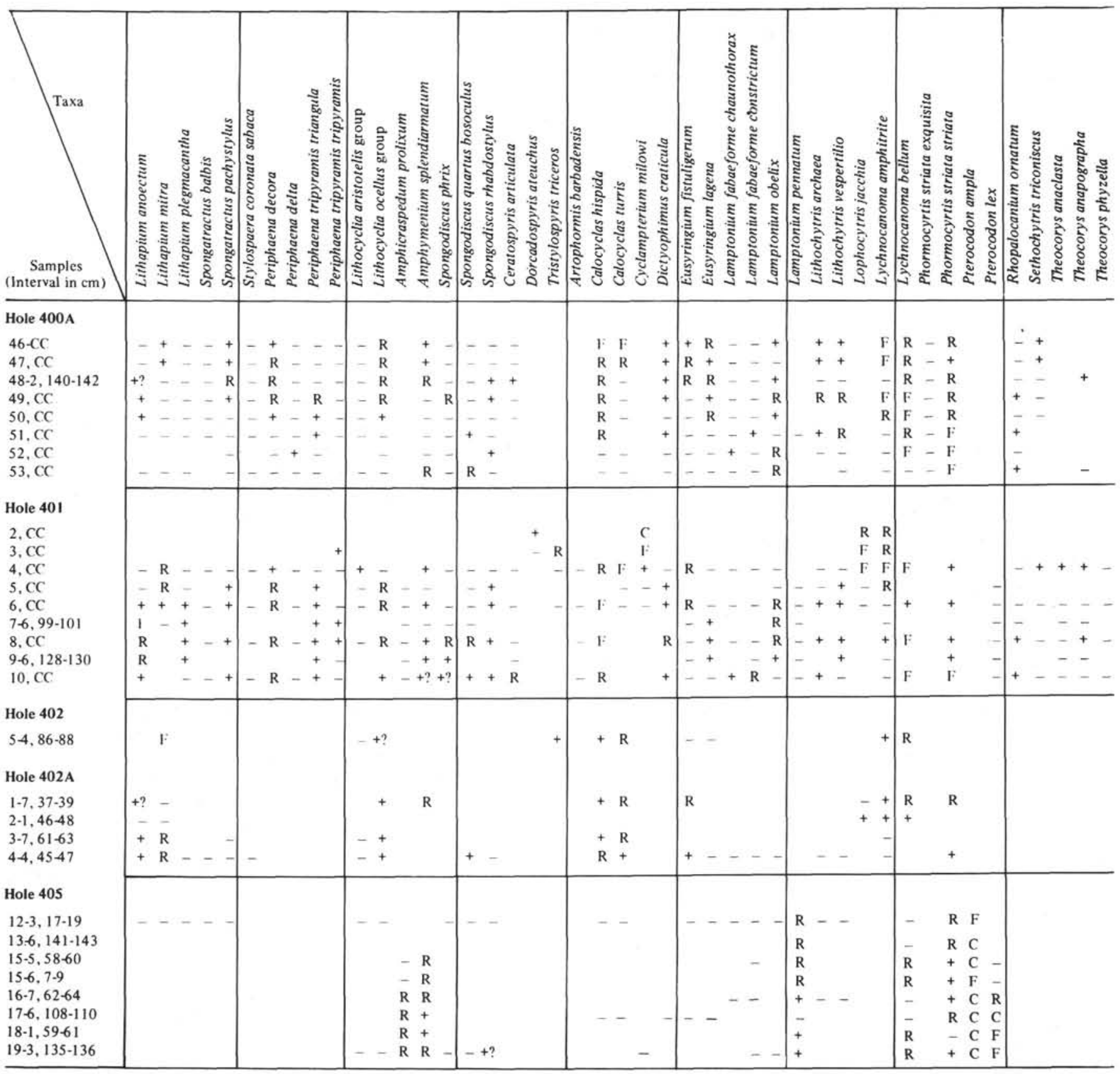

served, but are common enough to be tabulated (Table 2) and to be used in correlation (Figure 3),

The Paleocene assemblage in $400 \mathrm{~A}-59, \mathrm{CC}$ is not sufficiently well preserved for identification, and the same is true of the Cretaceous samples with the exception of 400A$64-3,76-78 \mathrm{~cm}$, which includes the Spongopyle insolita group (rare), Sphaerostylus lanceola (very rare), Stichomitra asymbatos group (rare), Theocorys antiqua (very rare), unidentified theoperid (pl. 1, fig. 11), similar to one occurring in the Coniacian-Santonian of Romania (Riedel and Sanfilippo 1974, pl. 15, fig. 6-10), Amphipyndax stocki (few). No pseudoaulophacids nor artostrobiids were found.

\section{Hole 401}

Few to common, moderately to well preserved radiolarians occur in middle and late Eocene cores, and in one Paleocene sample. Common radiolarians, not sufficiently well preserved to be useful, occur in early Eocene samples.

Middle and late Eocene species occurrences are shown in Table 2, and the correlations based on them are included in Figure 3. The Paleocene assemblage from 401-14-5, 93-95 $\mathrm{cm}$, includes Bekoma bidartensis (rare), B. campechensis (very rare), B. divaricata (very rare), Buryella tetradica (common), Lamptonium pennatum (rare), Phormocyrtis striata exquisita (few) and $P$. turgida (very rare). 


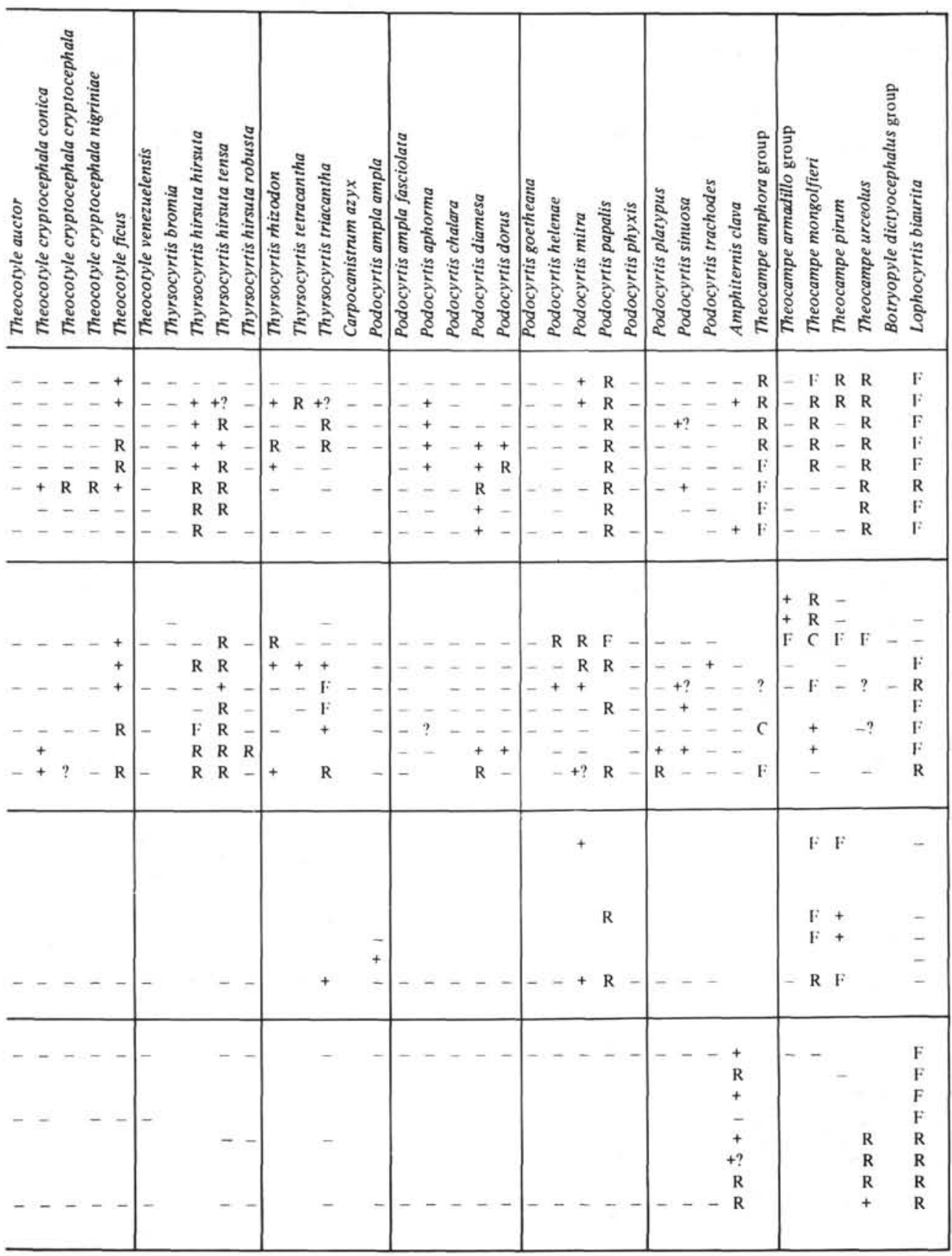

\section{Hole 402}

Few, well-preserved radiolarians occur in Core 1, but not in the other Quaternary cores. Few, moderately preserved radiolarians occur in the late Eocene Core 5 (Table 2 and Figure 3).

Forms occurring in the Quaternary Sample 402-1-4, $17-19 \mathrm{~cm}$ include Hexacontium hootsi (rare), Eucyrtidium cienkowskii group (rare), Litharachnium sp. (rare), Pterocorys sp. aff. $P$. hertwigii (very rare), Theocorythium sp. (rare), Artostrobium auritum group (rare), Carpocanarium spp. (rare). No saturnalins, artiscins, nor phacodiscids were encountered among the rather few specimens in the preparations.

\section{Hole 402A}

Few to common, moderately preserved radiolarians occur in the middle to late Eocene section. Rare, poorly preserved specimens occur in Albian samples from Cores 11-14, but not in samples examined from lower cores.

The assemblages occurring in Cores 402A-1 through -4 are sufficiently well preserved for taxonomic and stratigraphic interpretation, and the results shown in Table 2 and Figure 3.

\section{Hole 403}

Only one of three Quaternary samples examined contains rare, poorly preserved radiolarians. In early Pliocene and 
TABLE 3

Eocene Radiolarian Events at Sites 400, 401, 402, and 405

\begin{tabular}{|c|c|c|c|c|c|}
\hline & Events & $400 \mathrm{~A}$ & 401 & $402 \mathrm{~A}$ & 405 \\
\hline $\mathrm{Tm}$ & $\begin{array}{l}\text { Lophocyrtis } \\
\text { jacchia }\end{array}$ & & $\begin{array}{l}\text { Above } \\
-2, \text { CC M } \\
(94)\end{array}$ & & \\
\hline $\mathrm{Tm}$ & $\begin{array}{l}\text { Lychnocanoma } \\
\text { amphitrite }\end{array}$ & $\begin{array}{l}\text { Above } \\
-46, C C G \\
(512)\end{array}$ & $\begin{array}{l}\text { Above } \\
-2, \text { CC G } \\
(94)\end{array}$ & $\begin{array}{l}\text { Above } \\
-1-7 \mathrm{M} \\
(146)\end{array}$ & \\
\hline $\mathrm{Bm}$ & $\begin{array}{l}\text { Dorcadospyris } \\
\text { ateuchus }\end{array}$ & & $\begin{array}{l}-2, \mathrm{CC} \\
-3, \mathrm{CC} P \\
(94-104)\end{array}$ & & \\
\hline $\mathrm{Tm}$ & $\begin{array}{l}\text { Tristylospyris } \\
\text { triceros }\end{array}$ & & $\begin{array}{l}-3, \mathrm{CC} \\
-4, \mathrm{CC} \mathrm{P} \\
(104-113)\end{array}$ & & \\
\hline $\mathrm{Tm}$ & $\begin{array}{l}\text { Sethochytris } \\
\text { triconiscus }\end{array}$ & $\begin{array}{l}\text { Above } \\
46, C C M \\
(512)\end{array}$ & & & \\
\hline $\operatorname{Tm}$ & $\begin{array}{l}\text { Amphymenium } \\
\text { splendiarmatum }\end{array}$ & $\begin{array}{l}\text { Above } \\
46, \text { CC P } \\
(512)\end{array}$ & & & \\
\hline $\operatorname{Tm}$ & $\begin{array}{l}\text { Periphaena } \\
\text { decora }\end{array}$ & $\begin{array}{l}\text { Above } \\
-46, C C M \\
(512)\end{array}$ & $\begin{array}{l}\text { Above } \\
-4, \mathrm{CC} \mathrm{M} \\
(113)\end{array}$ & & \\
\hline $\mathrm{Tm}$ & $\begin{array}{l}\text { Theocampe } \\
\text { pinum }\end{array}$ & $\begin{array}{l}\text { Above } \\
-46, C C M \\
(512)\end{array}$ & $\begin{array}{l}-3, \mathrm{CC} \\
-4, \mathrm{CC} \mathrm{M} \\
(104-113)\end{array}$ & $\begin{array}{l}\text { Above } \\
-5-4 \mathrm{M} \\
(133)\end{array}$ & \\
\hline $\mathrm{Tm}$ & $\begin{array}{l}\text { Theocampe } \\
\text { mongol fieri }\end{array}$ & $\begin{array}{l}\text { Above } \\
-46, C C G \\
(512)\end{array}$ & $\begin{array}{l}\text { Above } \\
-4, C C \mathrm{G} \\
(113)\end{array}$ & & \\
\hline $\mathrm{Tm}$ & $\begin{array}{l}\text { Podocyrtis } \\
\text { helenae }\end{array}$ & & $\begin{array}{l}\text { Above } \\
-4, \mathrm{CC} \mathrm{M} \\
(113)\end{array}$ & & \\
\hline $\mathrm{Tm}$ & $\begin{array}{l}\text { Lithapium } \\
\text { mitra }\end{array}$ & $\begin{array}{l}\text { Above } \\
-46, C C G \\
(512)\end{array}$ & $\begin{array}{l}\text { Above } \\
-4, \text { CC G } \\
(113)\end{array}$ & $\begin{array}{l}\text { Above } \\
-1-7 \mathrm{P} \\
(146)\end{array}$ & \\
\hline $\operatorname{Tm}$ & $\begin{array}{l}\text { Eusyringium } \\
\text { fistuligerum }\end{array}$ & $\begin{array}{l}\text { Above } \\
-46, \mathrm{CC} \mathrm{M} \\
(512)\end{array}$ & $\begin{array}{l}\text { Above } \\
-4, C C \mathrm{G} \\
(113)\end{array}$ & $\begin{array}{l}\text { Above } \\
-1-7 \mathrm{G} \\
(146)\end{array}$ & \\
\hline $\mathrm{Tm}$ & $\begin{array}{l}\text { Calocyclas } \\
\text { hispida }\end{array}$ & $\begin{array}{l}\text { Above } \\
-46, C C G \\
(512)\end{array}$ & $\begin{array}{l}\text { Above } \\
-4, C C \mathrm{G} \\
(113)\end{array}$ & $\begin{array}{l}\text { Above } \\
-1-7 \mathrm{M} \\
(146)\end{array}$ & \\
\hline $\mathrm{Tm}$ & $\begin{array}{l}\text { Lychnocanoma } \\
\text { bellum }\end{array}$ & $\begin{array}{l}\text { Above } \\
-46, C C G \\
(512)\end{array}$ & $\begin{array}{l}\text { Above } \\
-4, C C \mathrm{G} \\
(113)\end{array}$ & $\begin{array}{l}\text { Above } \\
-1-7 \mathrm{G} \\
(146)\end{array}$ & \\
\hline $\mathrm{Tm}$ & $\begin{array}{l}\text { Podocyrtis } \\
\text { papalis }\end{array}$ & $\begin{array}{l}\text { Above } \\
-46, C C \mathrm{G} \\
(512)\end{array}$ & $\begin{array}{l}\text { Above } \\
-4, \mathrm{CC} \mathrm{G} \\
(113)\end{array}$ & $\begin{array}{l}\text { Above } \\
-4-4 \mathrm{G} \\
(170)\end{array}$ & \\
\hline $\mathrm{Tm}$ & $\begin{array}{l}\text { Calocyclas } \\
\text { turris }\end{array}$ & $\begin{array}{l}\text { Above } \\
-46, \mathrm{CC} \mathrm{G} \\
(512)\end{array}$ & $\begin{array}{l}\text { Above } \\
-4, \mathrm{CC} \mathrm{M} \\
(113)\end{array}$ & $\begin{array}{l}\text { Above } \\
-1-7 \mathrm{M} \\
(146)\end{array}$ & \\
\hline $\mathrm{Tm}$ & $\begin{array}{l}\text { Theocotyle } \\
\text { ficus }\end{array}$ & $\begin{array}{l}\text { Above } \\
-46, \text { CC P } \\
(512)\end{array}$ & $\begin{array}{l}\text { Above } \\
-4, C C P \\
(113)\end{array}$ & & \\
\hline $\mathrm{Tm}$ & $\begin{array}{l}\text { Phormocyrtis } \\
\text { striata striata }\end{array}$ & $\begin{array}{l}\text { Above } \\
-46, C \text { C P } \\
(512)\end{array}$ & $\begin{array}{l}\text { Above } \\
-4, C C M \\
(113)\end{array}$ & $\begin{array}{l}\text { Above } \\
-1-7 \mathrm{G} \\
(146)\end{array}$ & $\begin{array}{l}\text { Above } \\
-12-3 \mathrm{G} \\
(106)\end{array}$ \\
\hline $\mathrm{Tm}$ & $\begin{array}{l}\text { Dictyophimus } \\
\text { craticula }\end{array}$ & $\begin{array}{l}\text { Above } \\
-46, \mathrm{CC} \mathrm{M} \\
(512)\end{array}$ & $\begin{array}{l}-4, \mathrm{CC} \\
-5, \mathrm{CC} \mathrm{P} \\
(113-123)\end{array}$ & & \\
\hline $\mathrm{Bm}$ & $\begin{array}{l}\text { Cyclampterium } \\
\text { milowi }\end{array}$ & & $\begin{array}{l}-4, C C \\
-5, C C M \\
(113-123)\end{array}$ & & \\
\hline $\mathrm{Tm}$ & $\begin{array}{l}\text { Thyrsocyrtis } \\
\text { triacantha }\end{array}$ & & $\begin{array}{l}-4, \mathrm{CC} \\
-5, \mathrm{CC} \mathrm{M} \\
(113-123)\end{array}$ & & \\
\hline $\mathrm{Bm}$ & $\begin{array}{l}\text { Lophocyrtis } \\
\text { jacchia }\end{array}$ & & $\begin{array}{l}-4, C C \\
-5, C C G \\
(113-123)\end{array}$ & & \\
\hline $\mathrm{Tm}$ & $\begin{array}{l}\text { Lithocyclia } \\
\text { ocellus group }\end{array}$ & $\begin{array}{l}\text { Above } \\
-46, C C G \\
(512)\end{array}$ & $\begin{array}{l}-4, C C \\
-5, C C M \\
(113-123)\end{array}$ & $\begin{array}{l}\text { Above } \\
-1-7 \mathrm{M} \\
(146)\end{array}$ & \\
\hline
\end{tabular}

TABLE 3 - Continued

\begin{tabular}{|c|c|c|c|c|c|}
\hline & Events & $400 \mathrm{~A}$ & 401 & $402 \mathrm{~A}$ & 405 \\
\hline $\mathrm{Bm}$ & $\begin{array}{l}\text { Lithocyclia } \\
\text { aristotelis group }\end{array}$ & & $\begin{array}{l}4, \mathrm{CC} \\
-5, \mathrm{CC} M \\
(113-123)\end{array}$ & & \\
\hline $\operatorname{Tm}$ & $\begin{array}{l}\text { Lithochytris } \\
\text { vespertilio }\end{array}$ & $\begin{array}{l}\text { Above } \\
-46, C C M \\
(512)\end{array}$ & $\begin{array}{l}-4, \mathrm{CC} \\
-5, \mathrm{CC} \\
(113-123)\end{array}$ & & \\
\hline $\mathrm{Tm}$ & $\begin{array}{l}\text { Podocyrtis } \\
\text { mitra }\end{array}$ & $\begin{array}{l}\text { Above } \\
-46, \text { CC M } \\
(512)\end{array}$ & $\begin{array}{l}-4, C C \\
-5, C C P \\
(113-123)\end{array}$ & $\begin{array}{l}\text { Above } \\
4-4 \mathrm{M} \\
(170)\end{array}$ & \\
\hline $\operatorname{Tm}$ & $\begin{array}{l}\text { Podocyrtis } \\
\text { trachodes }\end{array}$ & & $\begin{array}{l}-4, C C \\
-5, C C \text { P } \\
(113-123)\end{array}$ & & \\
\hline $\mathrm{Tm}$ & $\begin{array}{l}\text { Lophocyrtis } \\
\text { biaurita }\end{array}$ & $\begin{array}{l}\text { Above } \\
-46, \mathrm{CC} \text { G } \\
(512)\end{array}$ & $\begin{array}{l}-4, \mathrm{CC} \\
-5, \mathrm{CC} M \\
(113-123)\end{array}$ & & $\begin{array}{l}\text { Above } \\
-12-3 \mathrm{G} \\
(106)\end{array}$ \\
\hline $\mathrm{Tm}$ & $\begin{array}{l}\text { Spongatractus } \\
\text { pachystylus }\end{array}$ & $\begin{array}{l}\text { Above } \\
-46, \text { CC M } \\
(512)\end{array}$ & $\begin{array}{l}-4, \text { CC } \\
-5, \text { CC P } \\
(113-123)\end{array}$ & & \\
\hline $\mathrm{Tm}$ & $\begin{array}{l}\text { Lithochytris } \\
\text { archaea }\end{array}$ & $\begin{array}{l}\text { Above } \\
46, \text { CC P } \\
(512)\end{array}$ & $\begin{array}{l}-4, \mathrm{CC} \\
-6, \mathrm{CC} \mathrm{P} \\
(113-132)\end{array}$ & & \\
\hline $\mathrm{Tm}$ & $\begin{array}{l}\text { Lamptonium } \\
\text { obelix }\end{array}$ & $\begin{array}{l}\text { Above } \\
-46, \text { CC P } \\
(512)\end{array}$ & $\begin{array}{l}-5, \mathrm{CC} \\
-6, \mathrm{CC} \mathrm{P} \\
(123-132)\end{array}$ & & \\
\hline $\mathrm{Tm}$ & $\begin{array}{l}\text { Eusyringium } \\
\text { lagena }\end{array}$ & $\begin{array}{l}\text { Above } \\
-46, \text { CC G } \\
(512)\end{array}$ & $\begin{array}{l}-6, \mathrm{CC} \\
-7-6 \quad \mathrm{M} \\
(132-141)\end{array}$ & & \\
\hline $\mathrm{Bm}$ & $\begin{array}{l}\text { Sethochytris } \\
\text { triconiscus }\end{array}$ & $\begin{array}{l}-47, \mathrm{CC} \\
-48-2 \mathrm{M} \\
(521-525)\end{array}$ & $\begin{array}{l}-4, C C \\
-6, C C \text { P } \\
(113-132)\end{array}$ & & \\
\hline $\mathrm{Bm}$ & $\begin{array}{l}\text { Theocampe } \\
\text { pirum }\end{array}$ & $\begin{array}{l}-47, \mathrm{CC} \\
-48-2 \quad \mathrm{M} \\
(521-525)\end{array}$ & $\begin{array}{l}-4, \mathrm{CC} \\
-5, \mathrm{CC} P \\
(113-123)\end{array}$ & & \\
\hline $\mathrm{Tm}$ & $\begin{array}{l}\text { Spongodiscus } \\
\text { rhabdostylus }\end{array}$ & $\begin{array}{l}-47, \mathrm{CC} \\
-48-2 \quad \mathrm{P} \\
(521-525)\end{array}$ & $\begin{array}{l}-4, C C \\
-5, C C P \\
(113-123)\end{array}$ & & \\
\hline $\operatorname{Tm}$ & $\begin{array}{l}\text { Lithapium } \\
\text { anoectum }\end{array}$ & $\begin{array}{l}-47, \mathrm{CC} \\
-48-2 \quad P \\
(521-525)\end{array}$ & $\begin{array}{l}-5, \mathrm{CC} \\
-6, \mathrm{CC} M \\
(123-132)\end{array}$ & $\begin{array}{l}-2-1 \\
-3-7 \mathrm{M} \\
(147-166)\end{array}$ & \\
\hline $\mathrm{Bm}$ & $\begin{array}{l}\text { Calocyclas } \\
\text { turris }\end{array}$ & $\begin{array}{l}-47, \mathrm{CC} \\
-48-2 \quad M \\
(521-525)\end{array}$ & $\begin{array}{l}-4, \mathrm{CC} \\
-5, \mathrm{CC} M \\
(113-123)\end{array}$ & $\begin{array}{l}\text { Below } \\
-4-4 \mathrm{M} \\
(170)\end{array}$ & \\
\hline $\mathrm{Tm}$ & $\begin{array}{l}\text { Lithapium } \\
\text { plegmacantha }\end{array}$ & & $\begin{array}{l}-5, \mathrm{CC} \\
-6, \mathrm{CC} \mathrm{P} \\
(123-132)\end{array}$ & & \\
\hline $\mathrm{Bm}$ & $\begin{array}{l}\text { Podocyrtis } \\
\text { trachodes }\end{array}$ & & $\begin{array}{l}-5, \mathrm{CC} \\
-6, \mathrm{CC} \mathrm{P} \\
(123-132)\end{array}$ & & \\
\hline $\mathrm{Bm}$ & $\begin{array}{l}\text { Lithapium } \\
\text { mitra }\end{array}$ & $\begin{array}{l}-47, \mathrm{CC} \\
-48-2 \quad \mathrm{M} \\
(521-525)\end{array}$ & $\begin{array}{l}-6, \mathrm{CC} \\
-7-6 \quad M \\
(132-140)\end{array}$ & $\begin{array}{l}\text { Below } \\
4-4 \mathrm{G} \\
(170)\end{array}$ & \\
\hline $\mathrm{Bm}$ & $\begin{array}{l}\text { Podocyrtis } \\
\text { helenae }\end{array}$ & & $\begin{array}{l}-6, \mathrm{CC} \\
-7-6 \quad P \\
(132-140)\end{array}$ & & \\
\hline $\mathrm{Bm}$ & $\begin{array}{l}\text { Podocyrtis } \\
\text { mitra }\end{array}$ & $\begin{array}{l}-47, \mathrm{CC} \\
-48-2 \quad M \\
(521-525)\end{array}$ & $\begin{array}{l}-6, \mathrm{CC} \\
-7-6 \quad M \\
(132-140)\end{array}$ & $\begin{array}{l}\text { Below } \\
-4-4 \mathrm{M} \\
(170)\end{array}$ & \\
\hline $\mathrm{Tm}$ & $\begin{array}{l}\text { Podocyrtis } \\
\text { ampla ampla }\end{array}$ & & & $\begin{array}{l}-2-1 \\
-3-7 \mathrm{P} \\
(147-165)\end{array}$ & \\
\hline $\mathrm{Tm}$ & $\begin{array}{l}\text { Periphaena } \\
\text { tripyramis } \\
\text { triangula }\end{array}$ & $\begin{array}{l}-48-2 \\
-49, \mathrm{CC} M \\
(525-540)\end{array}$ & $\begin{array}{l}-4, C C \\
-5, C C \text { P } \\
(113-123)\end{array}$ & & \\
\hline $\mathrm{Bm}$ & $\begin{array}{l}\text { Podocyrtis } \\
\text { ampla ampla }\end{array}$ & & & $\begin{array}{l}-3-7 \\
-4-4 P \\
(165-170)\end{array}$ & \\
\hline $\mathrm{Bm}$ & $\begin{array}{l}\text { Eusyringium } \\
\text { fistuligerum }\end{array}$ & $\begin{array}{l}-48-2 \\
-49, \mathrm{CC} \mathrm{G} \\
(525-540)\end{array}$ & $\begin{array}{l}-6, C C \\
-7-6 \text { G } \\
(132-140)\end{array}$ & $\begin{array}{l}\text { Below } \\
-4-4 \mathrm{M} \\
(170)\end{array}$ & \\
\hline
\end{tabular}


TABLE 3 - Continued

\begin{tabular}{|c|c|c|c|c|c|}
\hline & Events & $400 \mathrm{~A}$ & 401 & $402 \mathrm{~A}$ & 405 \\
\hline $\mathrm{Tm}$ & $\begin{array}{l}\text { Rhopalocanium } \\
\text { ornatum }\end{array}$ & & $\begin{array}{l}-7-6 \\
-8, \text { CC P } \\
(140-151)\end{array}$ & & \\
\hline $\mathrm{Tm}$ & $\begin{array}{l}\text { Spongodiscus } \\
\text { phrix }\end{array}$ & $\begin{array}{l}-48-2 \\
-49, \text { CC P } \\
(525-540)\end{array}$ & $\begin{array}{l}-7-6 \\
-8, \mathrm{CC} M \\
(140-151)\end{array}$ & & \\
\hline $\mathrm{Tm}$ & $\begin{array}{l}\text { Podocyrtis } \\
\text { diamesa }\end{array}$ & $\begin{array}{l}-48-2 \\
-49, \mathrm{CC} M \\
(525-540)\end{array}$ & $\begin{array}{l}-8, C C \\
-9-6 \quad M \\
(151-160)\end{array}$ & & \\
\hline $\mathrm{Tm}$ & $\begin{array}{l}\text { Podocyrtis } \\
\text { dorus }\end{array}$ & $\begin{array}{l}-48-2 \\
-49, \mathrm{CC} \mathrm{M} \\
(525-540)\end{array}$ & $\begin{array}{l}-8, \mathrm{CC} \\
-9-6 \quad \mathrm{M} \\
(151-160)\end{array}$ & & \\
\hline $\mathrm{Tm}$ & $\begin{array}{l}\text { Podocyrtis } \\
\text { platypus }\end{array}$ & & $\begin{array}{l}-8, \mathrm{CC} \\
-9-6 \quad \mathrm{M} \\
(151-160)\end{array}$ & & \\
\hline $\mathrm{Tm}$ & $\begin{array}{l}\text { Theocotyle } \\
\text { cryptocephala } \\
\text { conica }\end{array}$ & $\begin{array}{l}-50, \mathrm{CC} \\
-51, \mathrm{CC} \mathrm{P} \\
(550-559)\end{array}$ & $\begin{array}{l}-8, C C \\
-9-6 \quad M \\
(151-160)\end{array}$ & & \\
\hline $\mathrm{Bm}$ & $\begin{array}{l}\text { Spongodiscus } \\
\text { phrix }\end{array}$ & $\begin{array}{l}-49, \mathrm{CC} \\
-50, \mathrm{CC} \text { P } \\
(540-550)\end{array}$ & $\begin{array}{l}\text { Below } \\
-10, \text { CC P } \\
(170)\end{array}$ & & \\
\hline $\mathrm{Tm}$ & $\begin{array}{l}\text { Lamptonium } \\
\text { fabaeforme } \\
\text { constrictum }\end{array}$ & $\begin{array}{l}-50, \mathrm{CC} \\
-51, \mathrm{CC} P \\
(550-559)\end{array}$ & $\begin{array}{l}-9-6 \\
-10, \text { CC M } \\
(160-170)\end{array}$ & & \\
\hline $\mathrm{Bm}$ & $\begin{array}{l}\text { Eusyringium } \\
\text { lagena }\end{array}$ & $\begin{array}{l}-50, \mathrm{CC} \\
-51, \mathrm{CC} \mathrm{M} \\
(550-559)\end{array}$ & $\begin{array}{l}-9-6 \\
-10, \text { CC P } \\
(160-170)\end{array}$ & & \\
\hline $\mathrm{Bm}$ & $\begin{array}{l}\text { Podocyrtis } \\
\text { dorus }\end{array}$ & $\begin{array}{l}-50, \mathrm{CC} \\
-51, \mathrm{CC} \mathrm{M} \\
(550-559)\end{array}$ & $\begin{array}{l}-9-6 \\
-10, \mathrm{CC} P \\
(160-170)\end{array}$ & & \\
\hline $\mathrm{Bm}$ & $\begin{array}{l}\text { Theocampe } \\
\text { mongolfieri }\end{array}$ & $\begin{array}{l}-50, \mathrm{CC} \\
-51, \mathrm{CC} \mathrm{M} \\
(550-559)\end{array}$ & $\begin{array}{l}-9-6 \\
-10, \mathrm{CC} \mathrm{M} \\
(160-170)\end{array}$ & $\begin{array}{l}\text { Below } \\
-4-4 \mathrm{M} \\
(170)\end{array}$ & $\begin{array}{l}\text { Above } \\
-12-3 \text { P } \\
(106)\end{array}$ \\
\hline $\mathrm{Bm}$ & $\begin{array}{l}\text { Lithapium } \\
\text { plegmacantha }\end{array}$ & & $\begin{array}{l}-9-6 \\
-10, \mathrm{CC} \mathrm{P} \\
(160-170)\end{array}$ & & \\
\hline $\mathrm{Bm}$ & $\begin{array}{l}\text { Thyrsocyrtis } \\
\text { triacantha }\end{array}$ & $\begin{array}{l}-49, \mathrm{CC} \\
-50, \mathrm{CC} \mathrm{M} \\
(540-550)\end{array}$ & $\begin{array}{l}\text { Below } \\
-10, C C \text { G } \\
(170)\end{array}$ & & \\
\hline $\mathrm{Bm}$ & $\begin{array}{l}\text { Spongatractus } \\
\text { pachystylus }\end{array}$ & $\begin{array}{l}-49, \mathrm{CC} \\
-50, \mathrm{CC} \mathrm{M} \\
(540-550)\end{array}$ & $\begin{array}{l}\text { Below } \\
-10, \text { CC M } \\
(170)\end{array}$ & & \\
\hline $\mathrm{Bm}$ & $\begin{array}{l}\text { Periphaena } \\
\text { decora }\end{array}$ & $\begin{array}{l}-50, \mathrm{CC} \\
-51, \mathrm{CC} \mathrm{M} \\
(550-559)\end{array}$ & $\begin{array}{l}\text { Below } \\
-10, \text { CC M } \\
(170)\end{array}$ & & \\
\hline $\mathrm{Tm}$ & $\begin{array}{l}\text { Theocotyle } \\
\text { cryptocephala } \\
\text { cryptocephala }\end{array}$ & $\begin{array}{l}-50, \mathrm{CC} \\
-51, \mathrm{CC} \text { P } \\
(550-559)\end{array}$ & & & \\
\hline $\mathrm{Bm}$ & $\begin{array}{l}\text { Lithapium } \\
\text { anoectum }\end{array}$ & $\begin{array}{l}-50, \mathrm{CC} \\
-51, \mathrm{CC} \mathrm{M} \\
(550-559)\end{array}$ & $\begin{array}{l}\text { Below } \\
-10, \text { CC M } \\
(170)\end{array}$ & $\begin{array}{l}\text { Below } \\
-4-4 \mathrm{M} \\
(170)\end{array}$ & \\
\hline $\mathrm{Tm}$ & $\begin{array}{l}\text { Theocotyle } \\
\text { cryptocephala } \\
\text { nigriniae }\end{array}$ & $\begin{array}{l}-50, C C \\
-51, C C P \\
(550-559)\end{array}$ & & & \\
\hline $\mathrm{Bm}$ & $\begin{array}{l}\text { Lychnocanoma } \\
\text { amphitrite }\end{array}$ & $\begin{array}{l}-50, \mathrm{CC} \\
-51, \mathrm{CC} \mathrm{M} \\
(550-559)\end{array}$ & $\begin{array}{l}-8, \mathrm{CC} \\
-9-6 \quad \mathrm{P} \\
(151-160)\end{array}$ & & \\
\hline $\mathrm{Bm}$ & $\begin{array}{l}\text { Lithocyclia } \\
\text { ocellus group }\end{array}$ & $\begin{array}{l}-50, \mathrm{CC} \\
-51, \mathrm{CC} \mathrm{M} \\
(550-559)\end{array}$ & $\begin{array}{l}\text { Below } \\
-10, C C \text { G } \\
(170)\end{array}$ & $\begin{array}{l}\text { Below } \\
-4-4 \mathrm{M} \\
(170)\end{array}$ & \\
\hline $\mathrm{Bm}$ & $\begin{array}{l}\text { Lithochytris } \\
\text { vespertilio }\end{array}$ & $\begin{array}{l}-51, \mathrm{CC} \\
-52, \mathrm{CC} \text { G } \\
(559-569) \\
\end{array}$ & $\begin{array}{l}-9-6 \\
-10, \text { CC P } \\
(170) \\
\end{array}$ & & \\
\hline
\end{tabular}

TABLE 3 - Continued

\begin{tabular}{|c|c|c|c|c|c|}
\hline & Events & $400 \mathrm{~A}$ & 401 & $402 \mathrm{~A}$ & 405 \\
\hline $\mathrm{Bm}$ & $\begin{array}{l}\text { Dictyophimus } \\
\text { craticula }\end{array}$ & $\begin{array}{l}-51, \mathrm{CC} \\
-52, \mathrm{CC} \text { P } \\
(559-569)\end{array}$ & $\begin{array}{l}\text { Below } \\
-10, \text { CC M } \\
(170)\end{array}$ & & \\
\hline $\mathrm{Bm}$ & $\begin{array}{l}\text { Theocotyle } \\
\text { cryptocephala } \\
\text { nigriniae }\end{array}$ & $\begin{array}{l}-51, C C \\
-52, \text { CC P } \\
(559-569)\end{array}$ & & & \\
\hline $\mathrm{Bm}$ & $\begin{array}{l}\text { Theocotyle } \\
\text { cryptocephala } \\
\text { cryptocephala }\end{array}$ & $\begin{array}{l}-51, \mathrm{CC} \\
-52, \mathrm{CC} \text { P } \\
(559-569)\end{array}$ & & & \\
\hline $\mathrm{Bm}$ & $\begin{array}{l}\text { Theocotyle } \\
\text { cryptocephala } \\
\text { conica }\end{array}$ & $\begin{array}{l}-51, \mathrm{CC} \\
-52, \mathrm{CC} \mathrm{P} \\
(559-569)\end{array}$ & & & \\
\hline $\mathrm{Bm}$ & $\begin{array}{l}\text { Calocyclas } \\
\text { hispida }\end{array}$ & $\begin{array}{l}-51, \mathrm{CC} \\
-52, \mathrm{CC} \mathrm{M} \\
(559-569)\end{array}$ & $\begin{array}{l}\text { Below } \\
-10, \text { CC G } \\
(170)\end{array}$ & $\begin{array}{l}\text { Below } \\
-4-4 \mathrm{M} \\
(170)\end{array}$ & \\
\hline $\mathrm{Bm}$ & $\begin{array}{l}\text { Lamptonium } \\
\text { fabaeforme } \\
\text { constrictum }\end{array}$ & $\begin{array}{l}-51, \mathrm{CC} \\
-52, \mathrm{CC} \mathrm{P} \\
(559-569)\end{array}$ & $\begin{array}{l}\text { Below } \\
-10, \text { CC M } \\
(170)\end{array}$ & & \\
\hline $\mathrm{Bm}$ & $\begin{array}{l}\text { Periphaena } \\
\text { tripyramis } \\
\text { triangula }\end{array}$ & $\begin{array}{l}-51, \mathrm{CC} \\
-52, \mathrm{CC} \mathrm{P} \\
(559-569)\end{array}$ & & & \\
\hline $\mathrm{Bm}$ & $\begin{array}{l}\text { Lithochytris } \\
\text { archaea }\end{array}$ & $\begin{array}{l}-51, \mathrm{CC} \\
-52, \mathrm{CC} \mathrm{P} \\
(559-569)\end{array}$ & $\begin{array}{l}\text { Below } \\
-10, \text { CC P } \\
(170)\end{array}$ & & \\
\hline $\mathrm{Bm}$ & $\begin{array}{l}\text { Theocotyle } \\
\text { ficus }\end{array}$ & $\begin{array}{l}-51, \mathrm{CC} \\
-52, \mathrm{CC} \mathrm{M} \\
(559-569)\end{array}$ & $\begin{array}{l}\text { Below } \\
-10, \text { CC M } \\
(170)\end{array}$ & & \\
\hline $\mathrm{Bm}$ & $\begin{array}{l}\text { Lychnocanoma } \\
\text { bellum }\end{array}$ & $\begin{array}{l}-52, \mathrm{CC} \\
-53, \mathrm{CC} \mathrm{G} \\
(569-578)\end{array}$ & $\begin{array}{l}\text { Below } \\
-10, \mathrm{CC} \text { G } \\
(170)\end{array}$ & & \\
\hline $\mathrm{Bm}$ & $\begin{array}{l}\text { Spongodiscus } \\
\text { rhabdostylus }\end{array}$ & $\begin{array}{l}-52, \mathrm{CC} \\
-53, \mathrm{CC} \mathrm{P} \\
(569-578)\end{array}$ & $\begin{array}{l}\text { Below } \\
-10, \text { CC M } \\
(170)\end{array}$ & $\begin{array}{l}\text { Below } \\
-4-4 \mathrm{G} \\
(170)\end{array}$ & \\
\hline $\mathrm{Bm}$ & $\begin{array}{l}\text { Lamptonium } \\
\text { obelix }\end{array}$ & $\begin{array}{l}\text { Below } \\
-53 \text {,CC M } \\
(578)\end{array}$ & $\begin{array}{l}-9-6 \\
-10, \text { CC P } \\
(160-170)\end{array}$ & & \\
\hline $\mathrm{Bm}$ & $\begin{array}{l}\text { Podocyrtis } \\
\text { diamesa }\end{array}$ & $\begin{array}{l}\text { Below } \\
-53, \text { CC M } \\
(578)\end{array}$ & $\begin{array}{l}\text { Below } \\
-10, \text { CC G } \\
(170)\end{array}$ & & \\
\hline $\mathrm{Bm}$ & $\begin{array}{l}\text { Rhopalocanium } \\
\text { omatum }\end{array}$ & & $\begin{array}{l}\text { Below } \\
-10, \text { CC P } \\
(170)\end{array}$ & & \\
\hline $\mathrm{Bm}$ & $\begin{array}{l}\text { Podocyrtis } \\
\text { platypus }\end{array}$ & & $\begin{array}{l}\text { Below } \\
-10, \text { CC M } \\
(170)\end{array}$ & & \\
\hline $\mathrm{Bm}$ & $\begin{array}{l}\text { Phormocyrtis } \\
\text { striata striata }\end{array}$ & $\begin{array}{l}\text { Below } \\
-53, \text { CC G } \\
(578)\end{array}$ & $\begin{array}{l}\text { Below } \\
-10, \text { CC G } \\
(170)\end{array}$ & $\begin{array}{l}\text { Below } \\
-4-4 \mathrm{M} \\
(170)\end{array}$ & $\begin{array}{l}\text { Below } \\
-19-3 \mathrm{P} \\
(174)\end{array}$ \\
\hline Bm & $\begin{array}{l}\text { Amphymenium } \\
\text { splendiarmatum }\end{array}$ & $\begin{array}{l}\text { Below } \\
-53, \mathrm{CC} \mathrm{M} \\
(578)\end{array}$ & & & \\
\hline $\mathrm{Bm}$ & $\begin{array}{l}\text { Pterocodon } \\
\text { lex }\end{array}$ & & & & $\begin{array}{l}-15-6 \\
-16-7 \mathrm{M} \\
(139-150)\end{array}$ \\
\hline $\mathrm{Bm}$ & $\begin{array}{l}\text { Lophocyrtis } \\
\text { biaurita }\end{array}$ & $\begin{array}{l}\text { Below } \\
-53, \text { CC G } \\
(578)\end{array}$ & $\begin{array}{l}\text { Below } \\
-10, \text { CC G } \\
(170)\end{array}$ & & $\begin{array}{l}\text { Below } \\
-19-3 \text { G } \\
(174)\end{array}$ \\
\hline $\mathrm{Bm}$ & $\begin{array}{l}\text { Podocyrtis } \\
\text { papalis }\end{array}$ & $\begin{array}{l}\text { Below } \\
-53, \mathrm{CC} \text { G } \\
(578)\end{array}$ & & $\begin{array}{l}\text { Below } \\
-4-4 \quad G \\
(170)\end{array}$ & \\
\hline
\end{tabular}

Note: The arrangement and abbreviations conform to those that we have used previously (Sanfilippo and Riedel, 1973, p. 479), except that the limits indicated are morphotypic and not evolutionary. 


\begin{tabular}{|c|c|c|c|c|c|c|c|}
\hline \multirow{3}{*}{ Age } & \multicolumn{7}{|c|}{ Holes } \\
\hline & \multicolumn{4}{|c|}{ Bay of Biscay } & \multicolumn{3}{|c|}{ Rockall Plateau } \\
\hline & $400 \mathrm{~A}$ & 401 & 402 & $402 A$ & 403 & 404 & 405 \\
\hline Quaternary & & & $\begin{array}{l}\text { Core } 1 \\
\text { Few } \\
\text { Good }\end{array}$ & & $\begin{array}{l}\text { Core } 2 \\
\text { Rare } \\
\text { Poor }\end{array}$ & & \\
\hline Pliocene & & & & & $\begin{array}{l}\text { Cores 7-8 } \\
\text { Few } \\
\text { Moderate }\end{array}$ & & \\
\hline $\begin{array}{l}\text { Late } \\
\text { Miocene }\end{array}$ & $\begin{array}{l}\text { Cores 20-25 } \\
\text { Rare } \\
\text { Very poor. } \\
\text { moderate }\end{array}$ & & & & $\begin{array}{l}\text { Cores } 11.22 \\
\text { Rare- few } \\
\text { Very poor } \\
\text { good }\end{array}$ & & \\
\hline $\begin{array}{l}\text { Middle } \\
\text { Miocene }\end{array}$ & $\begin{array}{l}\text { Core } 29 \\
\text { Few } \\
\text { Very poor }\end{array}$ & & & & & & \\
\hline $\begin{array}{l}\text { Early } \\
\text { Miocene }\end{array}$ & $\begin{array}{l}\text { Cores } 38.40 \\
\text { Few - common } \\
\text { Very poor- } \\
\text { poor }\end{array}$ & & & & & & \\
\hline Oligocene & $\begin{array}{l}\text { Cores } 43.46 \\
\text { Rare - common } \\
\text { Very poor - } \\
\text { poor }\end{array}$ & & & & & & \\
\hline $\begin{array}{l}\text { Late } \\
\text { Eocene }\end{array}$ & & $\begin{array}{l}\text { Cores 2-4 } \\
\text { Few - common } \\
\text { Moderate - } \\
\text { good }\end{array}$ & $\begin{array}{l}\text { Core } 5 \\
\text { Few } \\
\text { Moderate }\end{array}$ & $\begin{array}{l}\text { Core } 1 \\
\text { Few } \\
\text { Moderate }\end{array}$ & & & \\
\hline $\begin{array}{l}\text { Middle } \\
\text { Eocene }\end{array}$ & $\begin{array}{l}\text { Cores } 47.52 \\
\text { Few- common } \\
\text { Poor }\end{array}$ & $\begin{array}{l}\text { Cores 5-10 } \\
\text { Common } \\
\text { Moderate - } \\
\text { good }\end{array}$ & & $\begin{array}{l}\text { Cores } 2-4 \\
\text { Few - common } \\
\text { Moderate }\end{array}$ & & & $\begin{array}{l}\text { Cores } 12 \cdot 14 \\
\text { Common } \\
\text { Moderate }\end{array}$ \\
\hline $\begin{array}{l}\text { Early } \\
\text { Eocene }\end{array}$ & $\begin{array}{l}\text { Core } 53 \\
\text { Common } \\
\text { Poor }\end{array}$ & $\begin{array}{l}\text { Cores } 11-12 \\
\text { Common } \\
\text { Very poor }\end{array}$ & & & & $\begin{array}{l}\text { Cores } 7.8 \\
\text { Few } \\
\text { Poor }\end{array}$ & $\begin{array}{l}\text { Core } 15 \\
\text { Common } \\
\text { Moderate }\end{array}$ \\
\hline Paleocene & $\begin{array}{l}\text { Core } 59 \\
\text { Common } \\
\text { Very poor }\end{array}$ & $\begin{array}{l}\text { Core } 14 \\
\text { Common } \\
\text { Moderate }\end{array}$ & & & & & \\
\hline Cretaceous & $\begin{array}{l}\text { Cores } 64.66,72 \\
\text { Rare - common } \\
\text { Very poor- } \\
\text { moderate }\end{array}$ & & & $\begin{array}{l}\text { Cores } 11-14 \\
\text { Rare } \\
\text { Very poor- } \\
\text { poor }\end{array}$ & & & \\
\hline
\end{tabular}

Figure 1. Summary of radiolarian occurrences, with notations on abundance (rare, few, or common) and preservation (good, moderate, poor, or very poor).

late Miocene samples radiolarians are rare to few, usually moderately to well preserved.

Most of the radiolarians in the sparse assemblage at 403-2-4, 12-14 cm are similar to those recorded from Core 402-1, Thecosphaera grecoi (rare), Heliodiscus asteriscus (rare), Eucyrtidium cienkowskii (very rare), Artostrobium miralestense (rare), but two differences were noted. This more northerly assemblage yielded two specimens of a robust phacodiscid, and the majority of the artostrobiids here belong to Artostrobium miralestense rather than to the more delicate Artostrobium auritum group.

Samples 403-3-3, 88-90 cm and 403-22-6, 87-89 cm contain rare poorly preserved specimens insufficient for identification. In the assemblages from 403-7-2, 102-104 cm; 403-11-4, 117-119 cm; 403-12-7, 41-43 cm; 403-14-2, $68-70 \mathrm{~cm}$; and $403-21-2,144-148 \mathrm{~cm}$ orosphaerid fragments are rare, collosphaerids rare, actinommids common, artiscins very rare, phacodiscids rare, spongodiscids common, pyloniids, litheliids and spyrids rare, theoperids few to common, carpocaniids rare, pterocorythids rare to few, artostrobiids few to common, cannobotrythids rare. Iden- tified forms in the above samples are: Hexacontium hootsi (rare), Hexalonche heracliti (very rare), Thecosphaera grecoi (rare), Heliodiscus asteriscus (rare, not found in 403-72), Eucyrtidium cienkowskii group (very few), Eucyrtidium punctatum group (rare to few, not found in 403-7-2), Stichocorys peregrina (rare to few, not found in 403-7-2), Pterocanium spp. (rare), Carpocanarium spp. (rare, not found in 403-7-2), Artostrobium auritum group (rare to common), Artostrobium miralestense (rare), Lithomitra lineata group (rare to common), Siphocampe corbula (rare to few, none found in 403-11-3 and 403-12-7), Botryopyle dictyocephalus group (rare). Haeckeliella inconstans (rare), was found only in 403-14-2, 68-70 cm. Lithomelissa campanulaeformis (rare) was found only in 403-12-7, 41-43 $\mathrm{cm}$. Sample 403-7-2, 102-104 cm contained, in addition, reworked Cyrtocapsella japonica (rare) and $C$. tetrapera (rare).

\section{Hole 404}

Early Eocene samples contain few radiolarians, poorly preserved. In the assemblages from Samples 404-7-2, 100- 


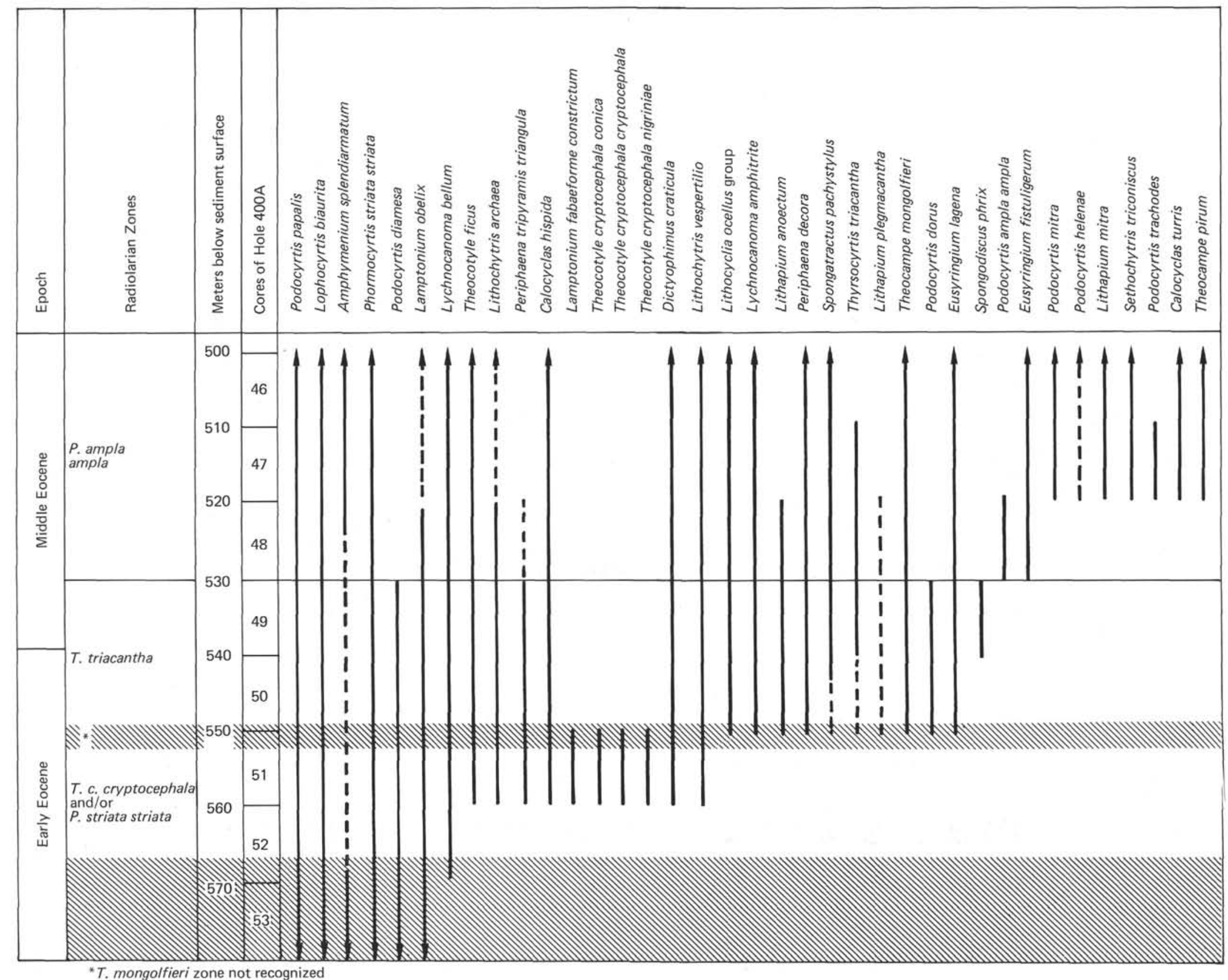




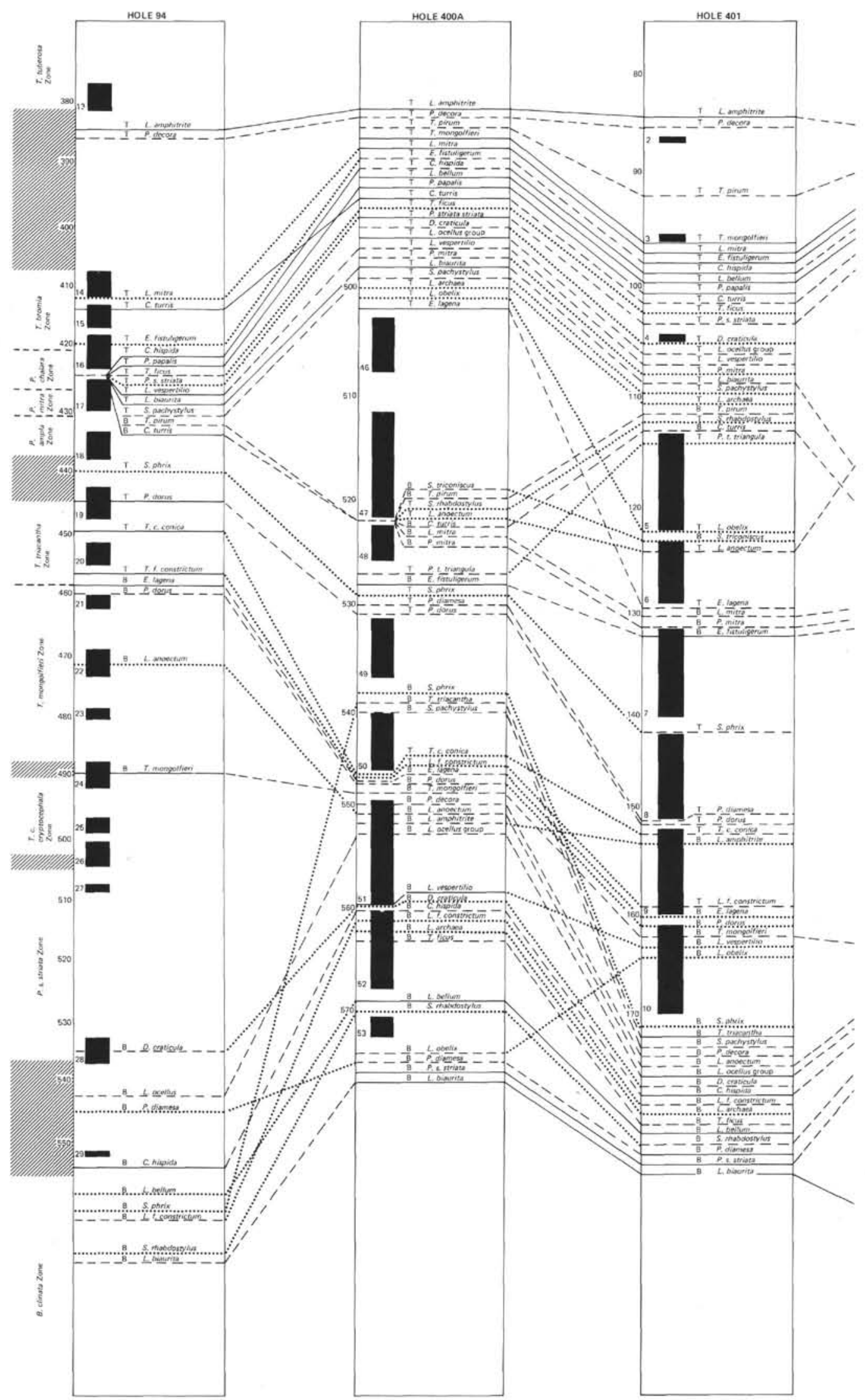

Figure 3. Radiolarian events available for correlation between Leg 48 holes, and with Hole 94 in the Gulf of Mexico (lefthand column). On the left of each column are shown depths below sediment surface in meters. Numbered black rectangles represent cores. Solid correlation lines are judged to be the most reliable, and dotted ones the least. The zonal boundaries at the left correspond to their positions in Hole 94. 


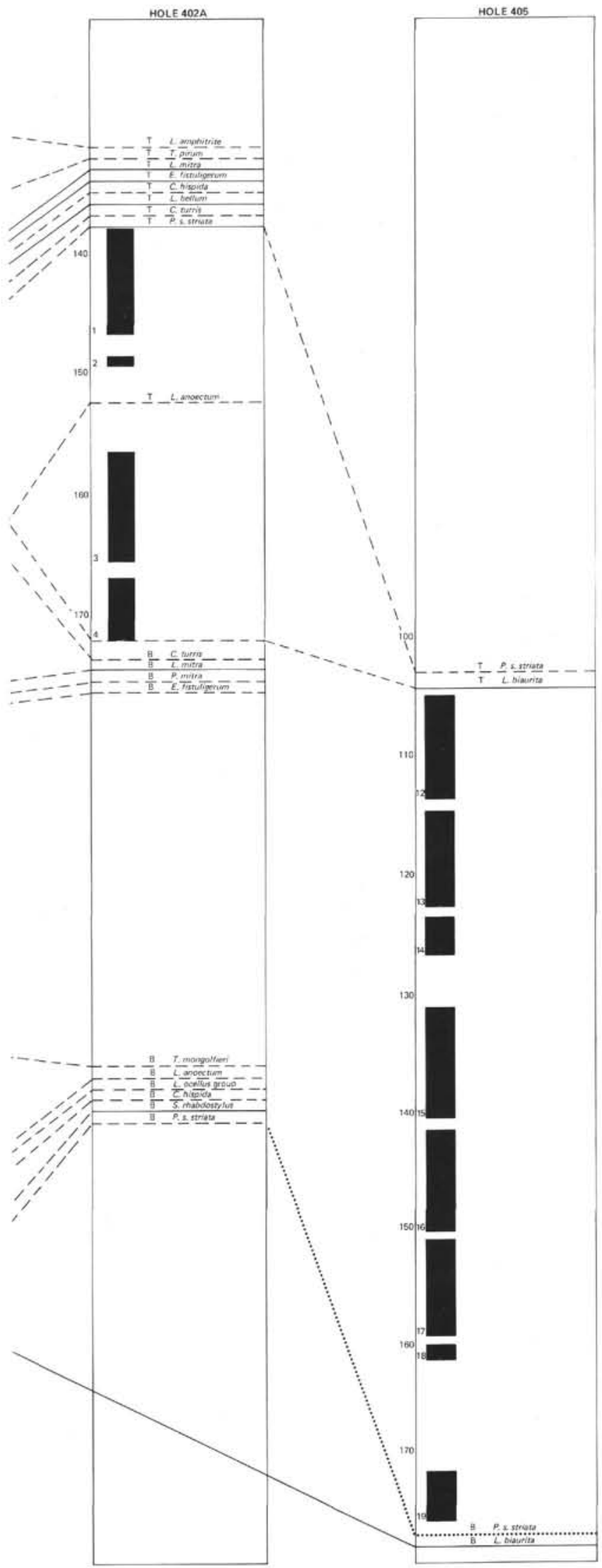

Figure 3. (Continued). 
$102 \mathrm{~cm} ; 404-8-1,128-131 \mathrm{~cm}$; and 404-8-2, $52-54 \mathrm{~cm}$, actinommids are common, spongodiscids few, phacodiscids few, spyrids moderately rare, theoperids rare, artostrobiids rare, and plagoniids rare.

\section{Hole 405}

In the eight samples examined from early and middle Eocene cores, radiolarians are common, and moderately preserved; their occurrences are shown in Table 2.

\section{STRATIGRAPHIC CORRELATION OF EOCENE SEQUENCES}

In Table 3, an attempt is made to arrange the earliest and latest occurrences of radiolarian taxa in stratigraphic order, with a minimum number of inconsistencies. The resulting range chart is shown in Figure 2, and the correlation diagram in Figure 3. Not all of the species included in Table 2 yielded data sufficiently useful to permit their incorporation in the events list, the range chart, or the correlation diagram.

In this initial investigation of the Leg 48 material we have concentrated on species useful for correlation in lower latitudes, with the consequence that some interesting forms that might be useful in higher latitudes have not been treated. The degree to which these North Atlantic sequences can be correlated with the low-latitude radiolarian zonation is indicated by the left-hand column of Figure 3, showing radiolarian events in the order of their occurrence at DSDP Site 94 in the Gulf of Mexico (Sanfilippo and Riedel, 1973). The boundaries between radiolarian zones are drawn to be consistent with definitions of zonal boundaries as revised by Riedel and Sanfilippo (in press).

As might be expected, correlations over this large latitudinal distance are tenuous, as they are to some extent between the sequences of widely spaced samples submitted. The Thyrsocyrtis bromia Zone seems to be represented by Cores 401-2 and -3 . Core 401-4 may belong either in the Podocyrtis chalara Zone or P. mitra Zone. The Podocyrtis ampla Zone may include Cores $400 \mathrm{~A}-46$ to $-48,401-5$ to -7 or -8 , and $402 \mathrm{~A}-1$ through -4 . The Thyrsocyrtis triacantha Zone seems to be represented by Cores $400 \mathrm{~A}-49$ and -50 , and 401-9 and possibly -8 . The Theocampe mongolfieri Zone was not recognized. Cores $400 \mathrm{~A}-51$, and possibly -52 and -53, and 401-10, apparently belong in the Theocotyle cryptocephala cryptocephala Zone or the Phormocyrtis striata striata Zone. It has not been possible to recognize any of these zones in the cores from Site 405 .

\section{SPECIES LIST}

The purpose of this list is to provide bibliographic references to the taxa mentioned in this chapter, and to serve as a taxonomic index. We have rather uncritically followed the generic assignments applied by earlier authors, since shortage of time has prevented our examining the relationships of type species of genera. Type specimens of new species will be deposited in the U.S. National Museum of Natural History.

\footnotetext{
Amphicraspedum prolixum Sanfilippo and Riedel

Amphicraspedum prolixum Sanfilippo and Riedel, 1973, p. 524, pl. 10, fig. $7-11$; pl. 28, fig. 3,4 .

This chapter: Table 2.
}

\section{Amphipyndax stocki (Campbell and Clark)}

Stichocapsa (?) stocki Campbell and Clark, 1944b, p. 44, pl. 8, fig. 31-33. Amphipyndx stocki (Campbell and Clark), Foreman, 1968, p. 78, pl. 8, fig. 12a-c.

This chapter: Site $400 \mathrm{~A}$ text.

\section{Amphiternis clava (Ehrenberg)}

Lithocampe ? clava Ehrenberg, 1873, p. 238; 1875, p. 76, pl. 4, fig. 2. Amphiternis clava (Ehrenberg), Foreman, 1973a, p. 430, pl. 7, fig. 16, 17; pl. 9, fig. 2, 7.

This chapter: Table 2.

\section{Amphymenium splendiarmatum Clark and Campbell}

Amphymenium splendiarmatum Clark and Campbell, 1942, p. 46, pl. 1, fig. 12,14 .

This chapter: Tables 2, 3; Figure 2.

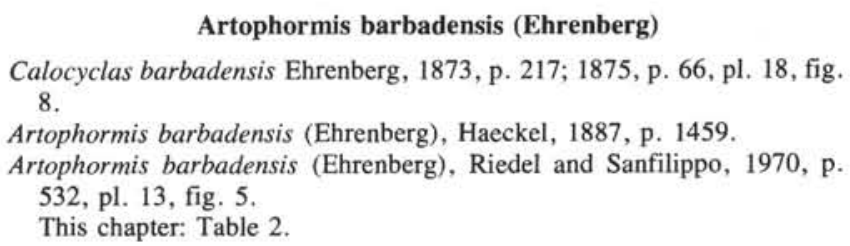

\section{Artostrobium auritum (Ehrenberg) group}

Lithocampe aurita Ehrenberg, 1844, p. 84; 1854, pl. 22, fig. 25.

Artostrobium auritum (Ehrenberg) group, Riedel and Sanfilippo, 1971, p. 1599, pl. $1 \mathrm{H}$, fig. 5-8.

This chapter: Site 402 text, 403 text.

\author{
Artostrobium miralestense (Campbell and Clark) \\ Dictyocephalus miralestensis Campbell and Clark, 1944a, p. 45, pl. 6, fig. \\ $12-14$. \\ Artostrobium miralestense (Campbell and Clark), Riedel and Sanfilippo, \\ 1971 , p. 1599 , pl. $1 \mathrm{H}$, fig. 9-17; pl. 2I, fig. 9, 10; pl. 3E, fig. 12. \\ This chapter: Site 403 text; Table 1.
}

\section{Bekoma bidartensis Riedel and Sanfilippo}

Bekoma bidartensis Riedel and Sanfilippo, 1971, p. 1592, pl. 7, fig. 1-7. Bekoma bidartensis Riedel and Sanfilippo, Foreman, 1973a, p. 432, pl. 3 , fig. 20, 21; pl. 10, fig. 6 .

This chapter: Site 401 text.

\section{Bekoma campechensis Foreman}

Bekoma campechensis Foreman, 1973a, p. 432, pl. 3, fig. 24; pl. 10, fig. $1,2,4$.

This chapter: Site 401 text.

\section{Bekoma divaricata Foreman}

Bekoma divaricata Foreman, 1973a, p. 433, pl. 3, fig. 23; pl. 10, fig. 3, 4. This chapter: Site 401 text.

\section{Botryopyle dictyocephalus Haeckel group}

Botryopyle dictyocephalus Haeckel, 1887, p. 1113, pl. 96, fig. 6.

Botryopyle dictyocephalus Haeckel group, Riedel and Sanfilippo, 1971, p. 1602, pl. 2J, fig. 20, 21; pl. 3F, fig. 13.

This chapter: Site 403 text.

\section{Buryella tetradica Foreman}

Buryella tetradica Foreman, 1973a, p. 433, pl. 8, fig. 4, 5; pl. 9, fig. 13, 14.

This chapter: Site 401 text.

\section{Calocyclas hispida (Ehrenberg)}

Anthocyrtis hispida Ehrenberg, 1873, p. 216; 1875, pl. 8, fig. 2.

Cycladophora hispida (Ehrenberg), Riedel and Sanfilippo, 1970, p. 529, pl. 10, fig. 9.

Calocyclas hispida (Ehrenberg), Foreman, 1973a, p. 434, pl. 1, fig. $12-15$; pl. 9, fig. 18.

This chapter: Tables 2, 3; Figures 2, 3 . 


\section{Calocyclas turris Ehrenberg}

Calocyclas turris Ehrenberg, 1873, p. 218; 1875, p. 66, pl. 18, fig. 7. Cycladophora turris (Ehrenberg), Riedel and Sanfilippo, 1970, p. 529, pl. 13 , fig. 3,4 .

Calocyclas turris Ehrenberg, Foreman, 1973a, p. 434

This chapter: Tables 2, 3; Figures 2, 3.

\section{Carpocanistrum (?) azyx Sanfilippo and Riedel}

Carpocanistrum (?) azyx Sanfilippo and Riedel, 1973, p. 530, pl. 35, fig. 9. This chapter: Table 2 .

\section{Ceratospyris articulata Ehrenberg}

Ceratospyris articulata Ehrenberg, 1873, p. 218; 1875, pl. 20, fig. 4. Ceratospyris articulata Ehrenberg, Sanfilippo and Riedel, 1973, p. 526, pl. 15, fig. 1-3; pl. 31, fig. 8,9 .

This chapter: Table 2.

\section{Cyclampterium leptetrum Sanfilippo and Riedel}

Cyclampterium ?leptetrum Sanfilippo and Riedel, 1970, p. 456, pl. 2, fig. $11,12$.

This chapter: Table 1.

\section{Cyclampterium milowi Riedel and Sanfilippo}

Cyclampterium (?) milowi Riedel and Sanfilippo, 1971, p. 1593, pl. 3B fig. 3; pl. 7, fig. 8, 9 .

This chapter: Tables 2,3 .

\section{Cyrtocapsella cornuta Haeckel}

Cyrtocapsa (Cyrtocapsella) cornuta Haeckel, 1887, p. 1513, pl. 78, fig. 9. Cyrtocapsella cornuta Haeckel, Sanfilippo and Riedel, 1970, p. 453, pl. 1 , fig. 19, 20.

This chapter: Table 1.

\section{Cyrtocapsella japonica (Nakaseko)}

Eusyringium japonicum Nakaseko, 1963, p. 193, pl. 4, fig. 1-3.

Cyrtocapsella japonica (Nakaseko), Sanfilippo and Riedel, 1970, p. 452, pl. 1, fig. 13-15.

This chapter: Site $400 \mathrm{~A}$ text, 403 text; Table 1.

\section{Cyrtocapsella tetrapera Haeckel}

Cyrtocapsa (Cyrtocapsella) tetrapera Haeckel, 1887, p. 1512, pl. 78, fig. 5.

Cyrtocapsella tetrapera Haeckel, Sanfilippo and Riedel, 1970, p. 453, pl. 1, fig. 16-18.

This chapter: Site 403 text; Table 1.

\section{Dictyophimus craticula Ehrenberg}

Dictyophimus craticula Ehrenberg, 1873, p. 223; 1875, pl. 5, fig. 4, 5. Dictyophimus craticula Ehrenberg, Sanfilippo and Riedel, 1973, p. 529, pl. 19 , fig. 1 ; pl. 33 , fig. 11 .

This chapter: Tables 2, 3; Figures 2, 3 .

\section{Dorcadospyris ateuchus (Ehrenberg)}

Ceratospyris ateuchus Ehrenberg, 1873, p. 218; 1875, pl. 21, fig. 4. Dorcadospyris ateuchus (Ehrenberg), Riedel and Sanfilippo, 1970, p. 523, pl. 15 , fig. 4 .

This chapter: Tables 2, 3 .

\section{Eucyrtidium cienkowskii Haeckel group}

cf. Eucyrtidium cienkowskii Haeckel, 1887, p. 1493, pl. 80, fig. 9. Eucyrtidium cienkowskii Haeckel group, Riedel and Sanfilippo in Sanfilippo et al., 1973, p. 221, pl. 5, fig. 5, 6 .

This chapter: Site 402 text, 403 text.

\section{Eucyrtidium diaphanes Sanfilippo and Riedel}

Calocyclas coronata Carnevale, 1908, p. 33, pl. 4, fig. 24 (not Eucyrtidium coronatum Ehrenberg, 1873).

Eucyrtidium diaphanes Sanfilippo and Riedel, in Sanfilippo et al., 1973 p. 221, pl. 5, fig. 12-14 (new name).

This chapter: Table 1.

\section{Eucyrtidium punctatum (Ehrenberg) group}

cf. Lithocampe punctata Ehrenberg, 1844, p. 84.

Eucyrtidium punctatum (Ehrenberg) group, Riedel and Sanfilippo, in Sanfilippo et al., 1973, p. 221, pl. 5, fig. 15, 16.

This chapter: Site 403 text.

\section{Eusyringium fistuligerum (Ehrenberg)}

Eucyrtidium fistuligerum Ehrenberg, 1873, p. 229; 1875, p. 70, pl. 9, fig.

Eusyringium fistuligerum (Ehrenberg), Riedel, 1957, p. 94, pl. 4, fig. 8. Eusyringium fistuligerum (Ehrenberg), Foreman 1973b, p. 435, pl. 11, fig. 6. This chapter: Tables 2, 3; Figures 2, 3

\section{Eusyringium lagena (Ehrenberg)}

Lithopera lagena Ehrenberg, 1873, p. 241, pl. 3, fig. 4.

Eusyringium lagena (Ehrenberg) (?), Riedel and Sanfilippo, 1970, p. 527, pl. 8 , fig. 5-7

Eusyringium lagena (Ehrenberg) (?), Foreman, 1973a, p. 435, pl. 11, fig. 4,5 .

This chapter: Tables 2, 3; Figures 2, 3.

\section{Haeckeliella inconstans Dumitrica}

Haeckeliella inconstans Dumitrica, 1973, p. 833, pl. 7, fig. 1, 2; pl. 18, fig. 7-22.

This chapter: Site 403 text.

\section{Heliodiscus asteriscus Haeckel}

Heliodiscus asteriscus Haeckel, 1887, p. 445, pl. 33, fig. 8 . This chapter: Site 403 text.

\section{Hexacontium hootsi Campbell and Clark}

Hexacontium hootsi Campbell and Clerk, 1944a, p. 14, pl. 2, fig. 5. This chapter: Site 402 text, 403 text.

\section{Hexalonche heracliti Haeckel}

Hexalonche heracliti Haeckel, 1887, p. 187, pl. 22, fig. 7. This chapter: Site 403 text.

\section{Lamptonium fabaeforme constrictum Riedel and Sanfilippo}

Lamptonium (?) fabaeforme (?) constrictum Riedel and Sanfilippo, 1970, p. 523 , pl. 5 , fig. 7.

This chapter: Tables 2, 3; Figures 2, 3.

Lamptonium fabaeforme chaunothorax Riedel and Sanfilippo

Lamptonium fabaeforme (?) chaunothorax Riedel and Sanfilippo, 1970, p. 524 , pl. 5, fig. 8,9 .

This chapter: Table 2 .

Lamptonium obelix Sanfilippo and Riedel, new species (Plate 1, Figures 1, 2)

Description: Shell inflated-ellipsoidal, not obviously three segmented. Thorax subspherical, with thick wall, rather smooth surface, and quincuncially arranged pores, comprising the bulk of the shell. Cephalis subspherical, not expressed in external contour, completely enclosed in the thick shell wall extending from the thorax to merge with the thick base of the apparently bladed apical horn. Abdomen truncate-conical, in some specimens much reduced, with narrow mouth, and pores less regular in size and arrangement than those of thorax. Lumbar stricture not pronounced externally. From near the base of the thorax, three short, stout-bladed wings project downward.

Measurements (based on 20 specimens from DSDP Samples 400A$52, \mathrm{CC} ; 400 \mathrm{~A}-53, \mathrm{CC}$; and $401-6, \mathrm{CC})$. Length overall, excluding horn, 155-290 $\mu \mathrm{m}$; cephalis and thorax, 105-165 $\mu \mathrm{m}$; greatest width of thorax $125-185 \mu \mathrm{m}$.

Remarks: This species is larger and more robust than Lamptonium pennatum, and its wings are situated more distally on the thorax. It shows some similarity to the form illustrated as Theocorys sp. aff. Theocorys spongoconum by Foreman (1973, pl. 11, fig. 14), but her illustration shows no horn nor wings.

We name it for the large companion of the Gallic hero Asterix. This chapter: Tables 2, 3; Figures 2, 3; Plate 1, Figures 1, 2. 


\section{Lamptonium pennatum Foreman}

Lamptonium pennatum Foreman, 1973a, p. 436, pl. 6, figs. 3-5, pl. 11, fig. 13 .

This chapter: Table 2; Plate 1, Figures 3, 4.

Lithapium anoectum Riedel and Sanfilippo

Lithapium anoectum Riedel and Sanfilippo, 1970, p. 520, pl. 4, fig. 4, 5. This chapter: Tables 2, 3; Figures 2, 3.

\section{Lithapium mitra (Ehrenberg)}

Cornutella mitra Ehrenberg, 1873, p. 221; 1875, pl. 2, fig. 8.

Lithapium (?) mitra (Ehrenberg) (?), Riedel and Sanfilippo, 1970, p. 520, pl. 4 , fig. 6,7 .

This chapter: Tables 2, 3; Figures 2, 3.

\section{Lithapium plegmacantha Riedel and Sanfilippo}

Lithapium (?) plegmacantha Riedel and Sanfilippo 1970, p. 520, pl. 4, fig. $2,3$.

Lithapium plegmacantha Riedel and Sanfilippo, Sanfilippo and Riedel, 1973, p. 516 , pl. 3 , fig. 1,2 , pl. 24 , fig. 8 , 9.

This chapter: Tables 2, 3; Figure 2.

\section{Lithochytris archaea Riedel and Sanfilippo}

Lithochytris archaea Riedel and Sanfilippo, 1970, p. 528, pl. 9, fig. 7. Lithochytris archaea Riedel and Sanfilippo, Foreman, 1973a, p. 436, pl 2 , fig. 4,5 .

This chapter: Tables 2, 3; Figures 2, 3 .

\section{Lithochytris vespertilio Ehrenberg}

Lithochytris vespertilio Ehrenberg, 1873, p. 239; 1875, pl. 4, fig. 10.

Lithochytris vespertilio Ehrenberg, Riedel and Sanfilippo, 1970, p. 528, pl. 9, fig. 8, 9 .

This chapter: Tables 2, 3; Figures 2, 3 .

\section{Lithocyclia aristotelis (Ehrenberg) group}

Astromma aristotelis Ehrenberg, 1847, p. 55, fig. 10.

Lithocyclia aristotelis (Ehrenberg) group, Riedel and Sanfilippo, 1970, p. $522 ; 1971$, p. 1588 , pl. $3 \mathrm{~A}$, fig. $4,5$.

This chapter: Tables 2,3 .

\section{Lithocyclia ocellus Ehrenberg group}

Lithocyclia ocellus Ehrenberg, 1854, pl. 36, fig. 30; 1873, p. 240.

Lithocyclia ocellus Ehrenberg group, Riedel and Sanfilippo, 1970, p. 522, pl. 5, fig. 1, 2 .

Lithocyclia ocellus Ehrenberg group, Sanfilippo and Riedel, 1973, p. 523, pl. 10 , fig. 1,2 .

This chapter: Tables 2, 3; Figures 2, 3.

\section{Lithomelissa campanulaeformis Campbell and Clark}

Lithomelissa campanulaeformis Campbell and Clark, 1944a, p. 41, pl. 6 , fig. 1 .

This chapter: Site 403 text.

\section{Lithomitra lineata (Ehrenberg) group}

Lithocampe lineata Ehrenberg, 1838, p. 130 (partim); 1854, pl. 22, fig. 26; pl. 36, fig. 16 .

Lithomitra lineata (Ehrenberg) group, Riedel and Sanfilippo, 1971, p. 1600 , pl. 1I, fig. 1-11; pl. 2I, fig. 14-16; pl. 3E, fig. 14

This chapter: Site 400 A text; Site 403 text; Table 1 .

\section{Lophocyrtis biaurita (Ehrenberg)}

Eucyrtidium biaurita Ehrenberg, 1873, p. 226; 1875, p. 70, pl. 10, fig. 7 , 8.

Lophocyrtis biaurita (Ehrenberg), Haeckel, 1887, p. 1411.

Lophocyrtis biaurita (Ehrenberg), Foreman, 1973a, p. 442, pl. 8, fig. 23-26.

This chapter: Tables 2, 3; Figures 2, 3 .

\section{Lophocyrtis jacchia (Ehrenberg)}

Thyrsocyrtis jacchia Ehrenberg, 1873, p. 261; 1875, pl. 12, fig. 7.

Lophocyrtis (?) jacchia (Ehrenberg), Riedel and Sanfilippo, 1970, p. 530

This chapter: Tables 2,3 .

\section{Lychnocanoma amphitrite Foreman}

Lychnocanoma amphitrite Foreman, 1973a, p. 437, pl. 11, fig. 10.

Remarks: In identifying specimens as belonging to this species, we have depended heavily on the size of the thorax (at least $150 \mu$ wide), in accordance with the original description. Rare specimens lack an abdomen. This chapter: Tables 2, 3; Figures 2, 3; Plate 1, Figures 5, 6.

\section{Lychnocanoma bellum (Clark and Campbell)}

Lychnocanium bellum Clark and Campbell, 1942, p. 72, pl. 9, fig. 35, 39. Lychnocanoma bellum (Clark and Campbell), Foreman, 1973a, p. 437, pl. 1 , fig. 17 ; pl. 11 , fig. 9 .

This chapter: Tables 2, 3; Figures 2, 3

\section{Periphaena decora Ehrenberg}

Periphaena decora Ehrenberg, 1873, p. 246; 1875, pl. 28, fig. 6 .

Periphaena decora Ehrenberg, Sanfilippo and Riedel, 1973, p. 523, pl. 8, fig. 8-10; pl. 27, fig. 2-5.

This chapter: Tables 2, 3; Figures 2, 3

\section{Periphaena delta Sanfilippo and Riedel}

Periphaena delta Sanfilippo and Riedel, 1973, p. 523, pl. 8, fig. 11, 12; pl. 27, fig. 6,7 .

This chapter: Table 2 .

\section{Periphaena tripyramis triangula (Sutton)}

Phacotriactus triangula Sutton, 1896, p. 61.

Triactus tripyramis triangula (Sutton), Riedel and Sanfilippo, 1970, p. 521 , pl. 4, fig. 9, 10.

Periphaena tripyramis triangula (Sutton), Sanfilippo and Riedel, 1973, p. 523 , pl. 9 , fig. 10,11

This chapter: Tables 2, 3; Figures 2, 3 .

\section{Periphaena tripyramis tripyramis (Haeckel)}

Triactus tripyramis Haeckel, 1887, p. 432, pl. 33, fig. 6.

Periphaena tripyramis tripyramis (Haeckel), Sanfilippo and Riedel, 1973, p. 523 , pl. 9, fig. 7-9.

This chapter: Table 2 .

\section{Phormocyrtis striata exqusita (Kozlova)}

Podocyrtis exquisita Kozlova, in Kozlova and Gorbovets, 1966, p. 106, pl. 17 , fig. 2

Phormocyrtis striata exquisita (Kozlova), Foreman, 1973a, p. 438, pl. 7 , fig. $1-4,7,8$; pl. 12 , fig. 5 .

This chapter: Table 2 .

\section{Phormocyrtis striata striata Brandt}

Phormocyrtis striata Brandt, in Wetzel, 1935, p. 55, pl. 9, fig. 12.

Phormocyrtis striata Brandt, Riedel and Sanfilippo, 1970, p. 532, pl. 10, fig. 7 .

Phormocyrtis striata striata Brandt, Foreman, 1973a, p. 438, pl. 7, fig. 5, $6,9$.

This chapter: Tables 2, 3; Figures 2, 3 .

\section{Podocyrtis ampla ampla Ehrenberg}

Podocyrtis ampla Ehrenberg, 1873, p. 248; 1875, pl. 16, fig. 7.

Podocyrtis ampla Ehrenberg, Riedel and Sanfilippo, 1970, p. 533, pl. 12, fig. 7,8 .

This chapter: Tables 2, 3; Figure 2

\section{Podocyrtis ampla fasciolata Nigrin}

Podocyrtis ampla fasciolata Nigrini, 1974, p. 1069, pl. 1K, fig. 1, 2; pl. 4, fig. 2,3 .

This chapter: Table 2 .

\section{Podocyrtis aphorma Riedel and Sanfilippo}

Podocyrtis aphorma Riedel and Sanfilippo, 1970, p. 534, pl. 11, fig. 2. This chapter: Table 2 .

\section{Podocyrtis chalara Riedel and Sanfilippo}

Podocyrtis chalara Riedel and Sanfilippo, 1970, p. 535, pl. 12, fig. 2, 3. This chapter: Table 2. 


\section{Podocyrtis diamesa Riedel and Sanfilippo}

Podocyrtis diamesa Riedel and Sanfilippo, 1970, p. 533, pl. 12, fig. 4-6. Podocyrtis diamesa Riedel and Sanfilippo, Sanfilippo and Riedel, 1973, p. 531 , pl. 20, fig. 9, 10; pl. 35, fig. 10, 11 .

This chapter: Tables 2, 3; Figures 2, 3 .

\section{Podocyrtis dorus Sanfilippo and Riedel}

Podocyrtis dorus Sanfilippo and Riedel, 1973, p. 531, pl. 35, fig. 12-14. This chapter: Tables 2, 3; Figures 2, 3.

\section{Podocyrtis goetheana (Haeckel)}

Cycladophora goetheana Haeckel, 1887, p. 1376, pl. 65, fig. 5 .

Podocyrtis goetheana (Haeckel), Riedel and Sanfilippo, 1970, p. 535. This chapter: Table 2.

\section{Podocyrtis helenae Nigrini}

Podocyrtis sp. B Riedel and Sanfilippo, 1973, p. 739, pl. 4, fig. 4-6.

Podocyrtis helenae Nigrini, 1974, p. 1070, pl. 1L, fig. 9-11; pl. 4, fig. 4, 5.

This chapter: Tables 2, 3; Figure 2.

\section{Podocyrtis mitra Ehrenberg}

Podocyrtis mitra Ehrenberg, 1854, pl. 36, fig. B20.

Podocyrtis mitra Ehrenberg, Riedel and Sanfilippo, 1970, p. 534, pl. 11, fig. 5,6 .

This chapter: Tables 2, 3; Figures 2, 3 .

\section{Podocyrtis papalis Ehrenberg}

Podocyrtis papalis Ehrenberg, 1847, fig. 2; 1854, pl. 36, fig. 23; 1873, p. 251.

Podocyrtis papalis Ehrenberg, Sanfilippo and Kiedel, 1973, p. 531, pl. 20. fig. 11-14; pl. 36, fig. 2, 3 .

This chapter: Tables 2, 3; Figures 2, 3 .

Podocyrtis phyxis Sanfilippo and Riedel

Podocyrtis phyxis Sanfilippo and Riedel, 1973, p. 531

This chapter: Table 2 .

\section{Podocyrtis platypus Sanfilippo and Riedel}

Podocyrtis platypus Sanfilippo and Riedel, 1973, p. 531, pl. 21, fig. 1-3; pl. 36 , fig. 4,5 .

This chapter: Tables 2, 3 .

\section{Podocyrtis sinuosa Ehrenberg}

[?] Podocyrtis sinuosa Ehrenberg, 1873, p. 253; 1875, pl. 15, fig. 5 . Podocyrtis sinuosa Ehrenberg (?), Riedel and Sanfilippo, 1970, p. 534, pl. 11 , fig. 3,4 .

This chapter: Table 2.

\section{Podocyrtis trachodes Riedel and Sanfilippo}

Podocyrtis trachodes Riedel and Sanfilippo, 1970, p. 535, pl. 11, fig. 7; pl. 12 , fig. 1 .

This chapter: Tables ;2, 3; Figure 2.

\section{Pterocodon ampla (Brandt)}

(?) Theocyrtis ampla Brandt, in Wetzel, 1935, p. 56, pl. 9, fig. 13-15. Pterocodon ampla (Brandt), Foreman 1973a, p. 438, pl. 5, fig. 3-5 This chapter: Table 2; Plate 1, Figures 7, 8 .

\section{Pterocodon lex Sanfilippo and Riedel, new species (Plate 1, Figures 9, 10)}

Description: Shell subcylindrical, of three segments, with slightly rough surface and ragged distal margin. Cephalis sub-hemispherical, with few pores, bearing a relatively long cylindro-conical horn. Collar stricture generally distinct. Thorax sub-hemispherical, with pores arranged approximately quincuncially. Lumbar stricture slightly, if at all, expressed externally, but marked internally by a distinct ring. Abdomen sub-cylindrical, with a few transferse rows of quincuncially arranged pores.

Measurements (based on 25 specimens from DSDP Samples 405-16-7, $62-64 \mathrm{~cm}$; 405-17-6, 108-110 cm; and 405-19-3, 135-136 cm). Length overall, excluding horn, 75-100 $\mu \mathrm{m}$; cephalis and thoras $45-80 \mu \mathrm{m}$; greatest width of thorax, $55-85 \mu \mathrm{m}$.
Remarks: This form is in many respects similar to Pterocodon (?) ampla Brandt (?) of Foreman (1973a, p. 438, pl. 5, fig. 3-5), but we do not synonymize them because her specimens have a substantially longer cephalis plus thorax and total length. Pterocodon lex is much smaller than the original specimens of Pterocodon ampla Brant, and is also much smaller than Theocorys unicum Lipman (in Lipman et al., 1960, p. 97, pl. 12, fig. 11) with which Foreman compared her specimens. As pointed out by Foreman, assignment to the genus Pterocodon is unsatisfactory, but we are unaware of a more appropriate genus, and reluctant to create a new one until more is known of the relationships of the species.

The name of the species is an arbitrary combination of letters, to be regarded as a feminine noun in apposition.

This chapter: Tables 2, 3; Plate 1, Figures 9, 10.

\section{Rhopalocanium ornatum Ehrenberg}

Rhopalocanium ornatum Ehrenberg, 1847, fig. 3; 1854, pl. 36, fig. 9: 1873 , p. $256 ; 1875$, p. 82 , pl. 17 , fig. 8 .

Rhopalocanium ornatum Ehrenberg, Foreman, 1973a, p. 439, pl. 2, fig $8-10$; pl. 12, fig. 3 .

This chapter: Tables 2, 3 .

\section{Sethochytris triconiscus Haeckel}

[?] Sethochytris triconiscus Haeckel, 1887, p. 1239, pl. 57, fig. 13.

Sethochytris triconiscus Haeckel (?), Riedel and Sanfilippo, 1970, p. 528, pl. 9, fig. 6.

This chapter: Tables 2, 3; Figures 2, 3 .

\section{Siphocampe corbula (Harting)}

Lithocampe corbula Harting, 1863, p. 12, pl. 1, fig. 21.

Siphocampe corbula (Harting), Nigrini, 1967, p. 85, pl. 8, fig. 5; pl. 9, fig. 3 .

This chapter: Hole $400 \mathrm{~A}$ text, 403 text.

\section{Sphaerostylus lanceola (Parona)}

Stylosphaera lanceola Parona, 1890, p. 150, pl. 1, fig. 19.

Sphaerostylus lanceola (Parona) group. Foreman, 1973b, p. 258, pl. 1, fig. 7-11.

This chapter: Hole 400A text.

\section{Spongatractus balbis Sanfilippo and Riedel}

Spongatractus balbis Sanfilippo and Riedel, 1973, p. 518, pl. 2, fig. 1-3; pl. 25 , fig. 1-2.

This chapter: Table 2 .

\section{Spongatractus pachystylus (Ehrenberg)}

Spongasphaera pachystyla Ehrenberg, 1873, p. 256; 1875, pl. 26, fig. 3. Spongatractus pachystylus (Ehrenberg), Haeckel, 1887, p. 350.

Spongatractus pachystylus (Ehrenberg), Sanfilippo and Riedel, 1973, p. 519 , pl. 2, fig. 4-6; pl. 25, fig. 3 .

This chapter: Tables 2, 3; Figures 2, 3.

\section{Spongodiscus quartus bosoculus Sanfilippo and Riedel}

Spongodiscus quartus bosoculus Sanfilippo and Riedel, 1973, p. 525, pl. 12, fig. $8-10$; pl. 29 , fig. 7 .

This chapter: Table 2 .

\section{Spongodiscus phrix Sanfilippo and Riedel}

Spongodiscus phrix Sanfilippo and Riedel, 1973, p. 525, pl. 12, fig. 1, 2; pl. 29 , fig. 2 .

This chapter: Tables 2, 3; Figures 2, 3.

\section{Spongodiscus rhabdostylus (Ehrenberg)}

Spongosphaera rhabdostyla Ehrenberg, 1873, p. 256; 1875, pl. 26, fig. 1, 2.

Spongodiscus rhabdostylus (Ehrenberg), Sanfilippo and Riedel, 1973, p. 525, pl. 13, fig. 1-3; pl. 30, fig. 1, 2.

This chapter: Tables 2, 3; Figure 3.

\section{Spongopyle insolita Kozlova group}

Spongopyle insolita Kozlova, in Kozlova and Gorbovets, 1966, p. 91, pl. 4, fig. 11a, b.

Spongopyle insolita Kozlova group, Riedel and Sanfilippo, 1974, p. 780, pl. 2, fig. 7-11; pl. 14, fig. 4

This chapter: Hole $400 \mathrm{~A}$ text. 
Stichocorys delmontensis (Campbell and Clark)

Eucyrtidium delmontense Campbell and Clark, 1944a, p. 56, pl. 7, fig. 19, 20.

Stichocorys delmontensis (Campbell and Clark), Sanfilippo and Riedel, 1970, p. 451, pl. 1, fig. 9; Riedel and Sanfilippo, 1971, p. 1595, pl. 1F, fig. 5-7; pl. 2E, fig. 10, 11 .

Stichocorys delmontensis (Campbell and Clark), Westberg and Riedel, in press.

This chapter: Hole $401 \mathrm{~A}$, Site 403 text.

\section{Stichocorys peregrina (Riedel)}

Eucyrtidium elongatum peregrinum Riedel, 1953, p. 812, pl. 85, fig. 2. Stichocorys peregrina (Riedel), Riedel and Sanfilippo, 1970, p. 530.

Stichocorys peregrina (Riedel), Westberg and Riedel, in press.

This chapter: Site 403 text.

\section{Stichomitra asymbatos Foreman group}

Stichomitra asymbatos Foreman, 1968, p. 73, pl. 8, fig. 10a-c.

Stichomitra asymbatos Foreman group, Riedel and Sanfilippo, 1974, p. 780 , pl. 10, fig. $1-7$; pl. 15 , fig. 5.

This chapter: Hole 400A text.

\section{Stylosphaera coronata sabaca Sanfilippo and Riedel}

Stylosphaera coronata sabaca Sanfilippo and Riedel, 1973, p. 521, pl. 1, fig. 18 ; pl. 25, fig. 7,8 .

This chapter: Table 2 .

\section{Tepka perforata Sanfilippo and Riedel}

Tepka perforata Sanfilippo and Riedel, in Sanfilippo et al., 1973, p. 228, pl. 6 , fig. 18-20.

This chapter: Table 1 .

\section{Thecosphaera grecoi Vinassa}

Thecosphaera grecoi Vinassa, 1900, p. 568, pl. 1, fig. 8.

This chapter: Site 403 text.

\section{Theocampe amphora (Haeckel) group}

Dictyocephalus amphora Haeckel, 1887, p. 1305, pl. 62, fig. 4.

Theocampe amphora (Haeckel) group, Foreman 1973a, p. 431, pl. 8, fig. 7, 9-13; pl. 9, fig. $8,9$.

This chapter: Table 2 .

\section{Theocampe armadillo (Ehrenberg) group}

Eucyrtidium armadillo Ehrenberg, 1873, p. 225; 1875, p. 70, pl. 9, fig. 10.

Theocampe armadillo (Ehrenberg) group, Riedel and Sanfilippo, 1971, p. 1601, pl. 3E, fig. 3-6.

This chapter: Table 2.

\section{Theocampe mongolfieri (Ehrenberg)}

Eucyrtidium mongolfiere Ehrenberg, 1854, pl. 36, fig. 18.

Theocampe mongolfieri (Ehrenberg), Burma, 1959, p. 329.

Theocampe mongolfieri (Ehrenberg), Riedel and Sanfilippo, 1970, p. 536, pl. 12 , fig. 9.

Theocampe mongolfieri (Ehrenberg), Foreman, 1973, p. 432, pl. 8, fig. 6, pl. 9, fig. 17 .

This chapter: Tables 2, 3; Figures 2, 3 .

\section{Theocampe pirum (Ehrenberg)}

Eucyrtidium pirum Ehrenberg, 1873, p. 232; 1875, p. 72, pl. 10, fig. 14.

Theocampe pirum (Ehrenberg), Riedel and Sanfilippo, 1971, p. 1601, pl. $3 \mathrm{E}$, fig. 10,11 .

This chapter: Tables 2, 3; Figures 2, 3 .

\section{Theocampe urceolus (Haeckel)}

Dictyocephalus urceolus Haeckel, 1887, p. 1305.

Theocampe urceolus (Haeckel), Foreman, 1973a, p. 432, pl. 8, fig. 14-17; pl. 9, fig. 6, 7 .

This chapter: Table 2 .

\section{Theocorys anaclasta Riedel and Sanfilippo}

Theocorys anaclasta Riedel and Sanfilippo, 1970, p. 530, pl. 10, fig. 2, 3. This chapter: Table 2 .

\section{Theocorys anapographa Riedel and Sanfilippo}

Theocorys anapographa Riedel and Sanfilippo, 1970, p. 530, pl. 10, fig. 4 This chapter: Table 2 .

Theocorys antiqua Squinabol

Theocorys antiqua Squinabol, 1903, p. 135, pl. 8, fig. 25. This chapter: Hole 400A text.

\section{Theocorys phyzella Foreman}

Theocorys (?) phyzella Foreman, 1973a, p. 440, pl. 5, fig. 8; pl. 12, fig. 1. This chapter: Table 2.

\section{Theocotyle auctor Foreman}

Theocotyle auctor Foreman, 1973a, p. 441, pl. 4, fig. 8-10, pl. 12, fig. 13. This chapter: Table 2 .

\section{Theocotyle cryptocephala conica Foreman}

Theocotyle cryptocephala conica Foreman, 1973a, p. 448, pl. 4, fig. 11; pl. 12, fig. 19, 20.

This chapter: Tables 2, 3; Figures 2, 3.

\section{Theocotyle cryptocephala cryptocephala (Ehrenberg)}

[?] Eucyrtidium cryptocephalum Ehrenberg, 1873, p. 227; 1875, p. 70, pl. 11 , fig. 11 .

Theocotyle cryptocephala cryptocephala (Ehrenberg) (?), Riedel and Sanfilippo, 1970 , p. 525 , pl. 6, fig. 7,8 .

This chapter: Tables 2, 3 .

\section{Theocotyle cryptocephala nigriniae Riedel and Sanfilippo}

Theocotyle cryptocephala (?) nigriniae Riedel and Sanfilippo, 1970, p. 525 , pl. 6, fig. 5,6 .

Theocotyle cryptocephala (?) nigriniae Riedel and Sanfilippo, Foreman, 1973 a, p. 440 , pl. 4 , fig. $1-5$; pl. 12, fig. 17.

This chapter: Tables 2, 3 .

\section{Theocotyle ficus (Ehrenberg)}

Eucyrtidium ficus Ehrenberg, 1873, p. 228; 1875, p. 70, pl. 11, fig. 19. Theocotyle ficus (Ehrenberg), Foreman, 1973a, p. 441, pl. 4, fig. 16-20. This chapter: Tables 2, 3; Figures 2, 3.

\section{Theocotyle venezuelensis Riedel and Sanfilippo}

Theocotyle venezuelensis Riedel and Sanfilippo, 1970, p. 525, pl. 6, fig. 9, 10; pl. 7, fig. 1, 2.

This chapter: Table 2 .

\section{Thyrsocyrtis bromia Ehrenberg}

Thyrsocyrtis bromia Ehrenberg, 1873, p. 260; 1875, p. 84, pl. 12, fig. 2.

Thyrsocyrtis bromia Ehrenberg, Riedel and Sanfilippo, 1970, p. 526; 1971, pl. 8, fig. 6 .

This chapter: Table 2 .

\section{Thyrsocyrtis hirsuta hirsuta (Krasheninnikov)}

Podocyrtis hirsutus Krasheninnikov, 1960, p. 300, pl. 3, fig. 16.

Thyrosocyrtis hirsuta hirsuta (Krasheninnikov), Riedel and Sanfillippo, 1970 , p. 526, pl. 7, fig. 9.

Thyrsocyrtis hirsuta hirsuta (Krasheninnikov), Foreman, 1973a, p. 441, pl. 3, fig. 3-8; pl. 12, fig. 15 .

This chapter: Table 2 .

\section{Thyrsocyrtis hirsuta robusta Riedel and Sanfilippo}

Thyrsocyrtis hirsuta robusta Riedel and Sanfilippo, 1970, p. 526, pl. 8, fig. 1 .

Thyrsocyrtis hirsuta robusta Riedel and Sanfilippo, Foreman, 1973a, p. 442, pl. 3, fig. 17.

This chapter: Table 2 .

\section{Thyrsocyrtis hirsuta tensa Foreman}

Thyrsocyrtis hirsuta tensa Foreman, 1973a, p. 442, pl. 3, fig. 13-16; pl. 12 , fig. 8 .

This chapter: Table 2 . 


\section{Thyrsocyrtis rhizodon Ehrenberg}

Thyrsocyrtis rhizodon Ehrenberg, 1873, p. 262; 1875, p. 94, pl. 12, fig. 1. Thyrsocyrtis rhizodon Ehrenberg, Foreman, 1973a, p. 442, pl. 3, fig. 1, 2. This chapter: Table 2.

\section{Thyrsocyrtis tetracantha (Ehrenberg)}

Podocyrtis tetracantha Ehrenberg, 1873, p. 254; 1875, p. 82, pl. 13, fig. 2.

Thyrsocyrtis tetracantha (Ehrenberg), Riedel and Sanfilippo, 1970, p. 527.

This chapter: Table 2 .

\section{Thyrsocyrtis triacantha (Ehrenberg)}

Podocyrtis triacantha Ehrenberg, 1873, p. 254; 1875, p. 82, pl. 13, fig. 4. Thyrsocyrtis triacantha (Ehrenberg), Riedel and Sanfilippo, 1970, p. 526, pl. 8, fig. 2, 3 .

Thyrsocyrtis triacantha (Ehrenberg), Foreman, 1973a, p. 442, pl. 12, fig. 9-11.

This chapter: Tables 2, 3; Figures 2, 3 .

\section{Tristylospyris triceros (Ehrenberg)}

Ceratospyris triceros Ehrenberg, 1873, p. 220; 1875, pl. 21, fig. 5. Tristylospyris triceros (Ehrenberg), Haeckel, 1887, p. 1033.

This chapter: Tables 2,3 .

\section{ACKNOWLEDGMENTS}

This research was supported by NSF Grant OCE75-20175. M.A. Hanger competently typed the manuscript, and assisted with proofreading.

\section{REFERENCES}

Brandt, R. In Wetzel, O., 1935. Die Mikropalaeontologie des Heiligenhafener Kieseltones (Ober-Eözan), Niedersaechs. Geol. Verhandl. Jahresber., v. 27, p. 41.

Burma, B.H., 1959. On the status of Theocampe Haeckel, and certain similar genera, Micropaleontology, v. 5, p. 325.

Campbell, A.S. and Clark, B.L., 1944a. Miocene radiolarian faunas from Southern California, Geol. Soc. Am., Spec. Paper, 51 .

1944b. Radiolaria from Upper Cretaceous of middle California, Geol. Soc. Am., Spec. Paper, 57.

Carnevale, P., 1908. Radiólarie e silicoflagellati di Bergonzano (Reggio Emilia), R. Ist. Veneto Sci. Lett. Arti, Mem., v. 28, p. 1.

Clark, B.L. and Campbell, A.S., 1942. Eocene radiolarian faunas from the Mt. Diablo area, California, Geol. Soc. Am., Spec. Paper, 39.

Dumitricà, P., 1973. Cretaceous and Quaternary Radiolaria in deep sea sediments from the northwest Atlantic Ocean and Mediterranean Sea. In Ryan, W.B.F., Hsü, K.J., et al., Initial Reports of the Deep Sea Drilling Project, v. 13: Washington (U.S. Government Printing Office), p. 829-901.

Ehrenberg, C.G., 1838. Über die Bildung der Kreidefelsen und des Kreidemergels durch unsichtbare Organismen, $\mathrm{Abh} . \mathrm{Kgl}$. Akad. Wiss. Berlin., Jahre 1838, p. 59.

1844. Über 2 neue Lager von Gebirgsmassen aus Infusorien als Meeres-Absatz in Nord-Amerika und eine Vergleichung derselben mit den organischen Kreide-Gebilden in Europa und Afrika, Kgl. Preuss. Akad. Wiss. Berlin, Ber., Jahre 1844 , p. 57-97.

, 1847. Über die mikroskopischen kieselschaligen Polycystinen als mächtige Gebirgsmasse von Barbados und über das Verhältniss der aus mehr als 300 Neuen Arten bestehenden ganz eigenthümlichen Formengruppe jener Felsmasse zu den jetzt lebenden Thieren und zur Kreidebildung. Eine neue An- regung zur Erforschung des Erdlebens, Kgl. Preuss. Akad. Wiss. Berlin, Ber., Jahre 1847, p. 40. 1854. Mikrogeologie: Leipzig (Voss). 1873. Grössere Felsproben des Polycystinen-Mergels von Barbados mit weiteren Erläuterungen. Monatsber, $\mathrm{Kgl}$. Preuss. Akad. Wiss. Berlin, Jahre 1873, p. 213. 1875. Fortsetzung der mikrogeologischen Studien als Gesammt-Uebersicht der mikroskopischen Paläontologie gleichartig analysirter Gebirgsarten der Erde, mit specieller Rücksicht auf den Polycystinen-Mergel von Barados, Abh. Kgl. Akad. Wiss. Berlin, Jahre 1975, p. 1.

Foreman, H.P., 1968. Upper Maestrichtian Radiolaria of California, Palaeontol. Assoc., London, Spec. Paper, 3, p. 1-82.

1973a, Radiolaria of Leg 10 with systematics and ranges for the families Amphipyndacidae, Artostrobiidae, and Theoperidae. In Worzel, J.L., Bryant, W., et al., Initial Reports of the Deep Sea Drilling Project, v. 10: Washington (U.S. Government Printing Office), p. 407-474. 1973b. Radiolaria from DSDP Leg 20. In Heezen, B.C., MacGregor, I.D., et al., Initial Reports of the Deep Sea Drilling Project, v. 20: Washington (U.S. Government Printing Office), p. 249-305.

Haeckel, E., 1887. Report on the Radiolaria collected by H.M.S. Challenger during the years 1873-76, Rept. Voyage Challenger, Zool., v. 18, p. 1-1803.

Harting, P., 1863. Bijdrage tot de kennis der mikroskopische faune en flora van de Banda-Zee, Kon. Akad. Wetensch. Amsterdam, Verh. 10. p. 1.

Kozlova, G.E. and Gorbovets, A.N., 1966. Radiolyarii verkhnemelovykh i verkhneeotsenovykh otlozhnenii ZapadnoSibirskoi Nizmennosti, Tr. Vses. Neft. Nauch.-Issled. Geologorazved. Inst. (VNIGRI), no. 248, p. 1-159.

Krasheninnikov, V.A., 1960. Nekotorye Radiolyarii Nizhnego i Srednego Eotsena Zapadnogo Predkavkazya, Min. Geol. $i$ Okhr. Nedr SSSR, Vses. Nauch. Issled. Geol. Neft. Inst., no. 16 , p. $1-271$.

Lipman, R. Kh., Burtman, E.S., and Khokhlova, I.A., 1960. Stratigraphy and fauna of the Paleogene deposits in the Western Siberian Lowland, Min. Geol. i Okhr. Nedr SSSR, Tr. Vses. Nauch.-Issled. Geol. Inst., new ser. 28, p. 69-98, 154-172.

Nakaseko, K., 1963. Neogene Cyrtoidea (Radiolaria) from the Isozaki Formation in Ibaraki Prefecture, Japan, Osaka Univ. Sci. Rept., v. 12, p. 165.

Nigrini, C., 1967. Radiolaria in pelagic sediments from the Indian and Atlantic oceans, Scripps Inst. Oceanog. Bull., Univ. Calif., v. 11 , p. $1-125$.

1974. Cenozoic Radiolaria from the Arabian Sea, DSDP Leg 23. In Whitmarsh, R.B., Weser, O.E., Ross, D.A., et al. Initial Reports of the Deep Sea Drilling Project, v. 23: Washington (U.S. Government Printing Office), p. 1051-1121.

Parona, C.F., 1890. Radiolarie nei noduli selciosi del calcare giurese di Cittiglio presso Laveno, Soc. Geol. Ital. Boll., v. 9, no. 1, p. 1-46.

Riedel, W.R., 1953. Mesozoic and late Tertiary Radiolaria of Rotti, J. Paleontol. v. 27, no. 6, p. 805-813.

1957. Radiolaria: a preliminary stratigraphy, Rept. Swedish Deep-Sea Exped., v. 6, p. 59.

1959. Oligocene and Lower Miocene Radiolaria in tropical Pacific sediments, Micropaleontology, v. 5, p. 285.

Riedel, W.R. and Foreman, H.P., 1961. Type specimens of North American Paleozoic Radiolaria, J. Paleontol., v. 35, no. 3, p. 628-632.

Riedel, W.R. and Sanfilippo, A., 1970. Radiolaria, Leg 4, Deep Sea Drilling Project. In Bader, R.G., Gerard, R.D., et al., Initial Reports of the Deep Sea Drilling Project, v. 4: Washington (U.S. Government Printing Office), p. 503-575. 
, 1971. Cenozoic Radiolaria from the western tropical Pacific, Leg 7. In Winterer, E.L., Riedel, W.R., et al., Initial Reports of the Deep Sea Drilling Project, v. 7: Washington (U.S. Government Printing Office), p. 1529-1672.

, 1973. Cenozoic Radiolaria from the Caribbean DSDP Leg 15. In Edgar, N.T., Saunders, J.B., et al., Initial Reports of the Deep Sea Drilling Project, v. 15: Washington (U.S. Government Printing Office), p. 705-751.

, 1974. Radiolaria from the southern Indian Ocean, DSDP Leg 26. In Davies, T.A., Luyendyk, B.P., et al., Initial Reports of the Deep Sea Drilling Project, v. 26: Washington (U.S. Government Printing Office), p. 771-814.

, in press. Stratigraphy and evolution of tropical Cenozoic radiolarians.

Sanfilippo, A., and Riedel, W.R., 1970. Post-Eocene "closed" theoperid radiolarians, Micropaleontology, v. 16, p. 446.

, 1973. Cenozoic Radiolaria (exclusive of theoperids, artostrobiids and amphipyndacids) from the Gulf of Mexico, DSDP Leg 10. In Worzel, J.L., Bryant, W., et al., Initial Reports of the Deep Sea Drilling Project, v. 10: Washington (U.S. Government Printing Office), p. 475-611.
1974. Radiolaria from the West-Central Indian Ocean and Gulf of Aden, DSDP Leg 24. In Fisher, R.L., Bunce, E.T., et al., Initial Reports of the Deep Sea Drilling Project, v. 24: Washington (U.S. Government Printing Office), 997-1035. , 1976. Radiolarian occurrences in the Caribbean Region. Publication de la VII Conference geologique des Caraibes du 30 juin au 12 juillet 1974, published in 1976 by VII Conference Geologique des Caraibes, Cayenne, Departement Francais de la Guyane, p. 145-168.

Sanfilippo, A., Burckle, L.H., Martini, E., and Riedel, W.R. 1973. Radiolarians, diatoms, silicoflagellates and calcareous nannofossils in the Mediterranean Neogene, Micropaleontology, v. 19 , no. 2, p. 209-234.

Sutton, H.J., 1896. Radiolaria; a new genus from Barbados, Am. Mon. Microsc. J., v. 17, p. 61.

Squinabol, S., 1903. Le Radiolarie di noduli selciosi nella Scaglia degli Euganei, Contrib. I. Riv. Ital. Paleontol., v. 9, p. 105 150.

Vinassa De Regny, P.E., 1900. Radiolaria miocenici italiani, Mem. R. Acc. Sci. Ist. Bologna, v. 8, p. 565-595.

Westberg, M.J. and Riedel, W.R., in press. Accuracy of Radiolarian correlations in the Pacific Miocene. 



\section{PLATE 1}

In the explanations to the figures, the sample numbers and slide designations (in the form "Ph.2," "Sl.2," "Cs.2," etc.) indicate preparations in our collection at Scripps Institution of Oceanography, and designations in the form " $\mathrm{R} 45 / 1$ " indicate England Finder positions of the illustrated specimens on the slides (Riedel and Foreman, 1961).

Figures 1, 2 Lamptonium obelix n. sp.

1. Holotype, DSDP 400A-52, CC, Ph. 1, Q28/0 $(190 \times)$.

2. Sample 401-6, CC, Cs.2, 040/4 (190×).

Figures 3, 4 Lamptonium pennatum.

3. Sample 405-13-6, 141-143 cm, S1.3, K39/4 $(280 \times)$.

4. Sample 405-12-3, 17-19 cm, Ph. 1, L19/2 $(280 \times)$.

Figures 5, $6 \quad$ Lychnocanoma amphitrite.

5. Sample 401-5, CC, Cs.1, T50/4 (190×).

6. Sample 401-5, CC, Cs.1, T40/3 (190×).

Figures 7, $8 \quad$ Pterocodon ampla.

7. Sample 405-19-3, 135-136 cm, Ph. 1, W29/0 $(280 \times)$.

8. Sample 405-18-1, 59-61 cm, Ph. 1, F14/4 $(280 \times)$.

Figures 9, $10 \quad$ Pterocodon lex $\mathrm{n}$. sp.

9. Holotype, Sample 405-17-6, 108-110 cm, F.1, Z43/3 (280x).

10. Sample $405-19-3,135-136 \mathrm{~cm}$, F. $1, \mathrm{Q} 40 / 0$ $(280 \times)$.

Figure 11 Unidentified theoperid similar to one occurring in the Coniacian-Santonian of Romania and Sample 25812-2. Sample 400A-64-3, 76-78 cm, Sl.1, Z17/0 $(280 \times)$.

Figure 12 Unidentified theoperid similar to one occurring in the Podocyrtis mitra Zone at DSDP Site 237 (San filippo and Riedel, 1974, pl. 3, fig. 5, 6), with unrecorded occurrences in the Thyrsocyrtis bromia Zone of Sample 162-4-6, 35-37 cm, and Sample WRTR-55 from Barbados (Sanfilippo and Riedel, 1976, p. 155). Sample 401-4, CC, Ph. 2, G17/1 (280×). 
PLATE 1
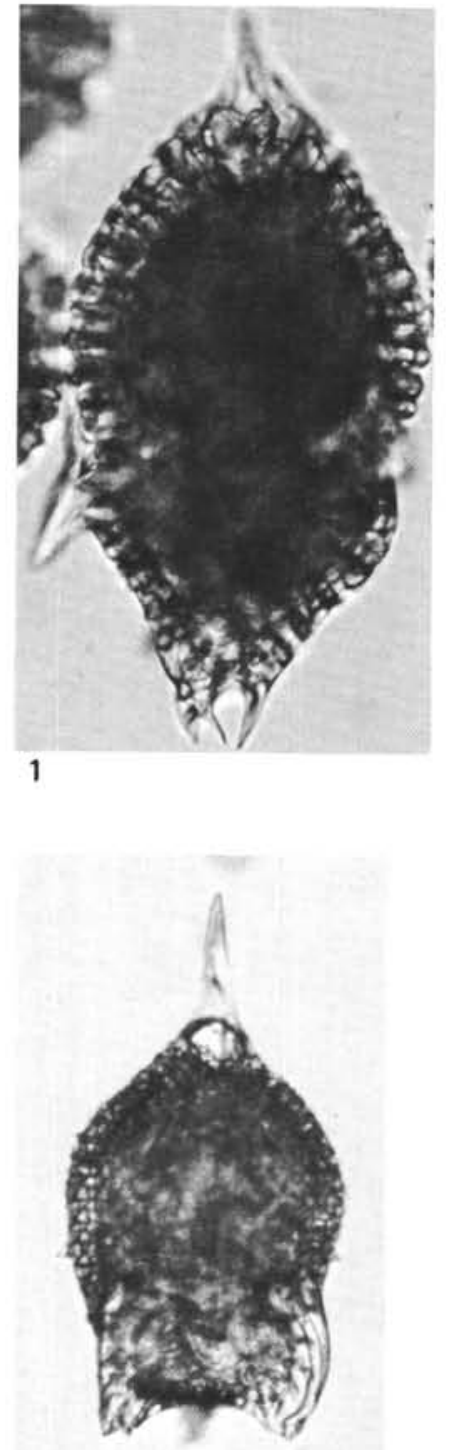

5
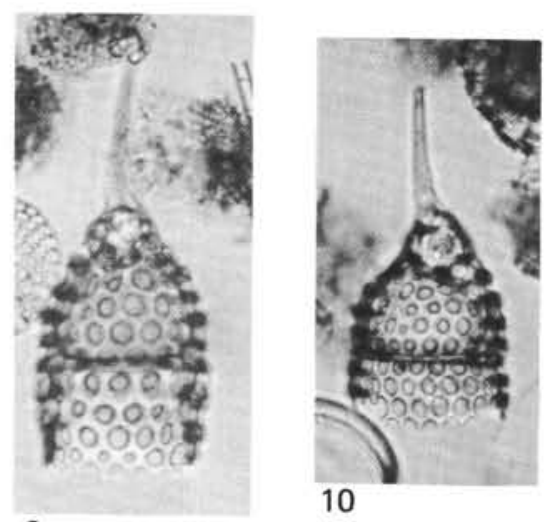

9

2

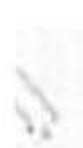

6
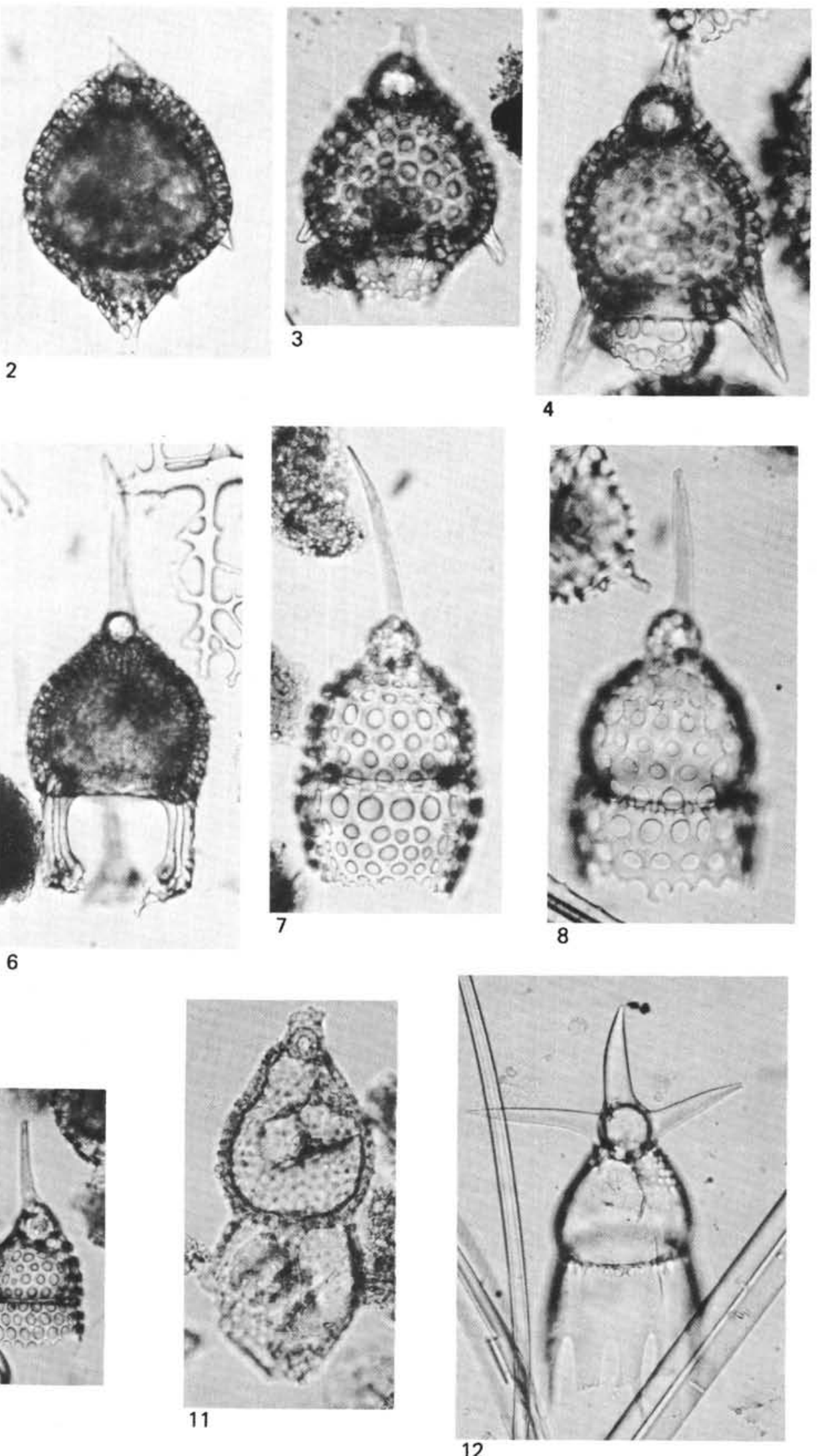